\title{
SPINORIALITY OF ORTHOGONAL REPRESENTATIONS OF REDUCTIVE GROUPS
}

\author{
ROHIT JOSHI AND STEVEN SPALLONE
}

Abstract. Let $G$ be a connected reductive group over a field $F$ of characteristic 0 , and $\varphi: G \rightarrow \mathrm{SO}(V)$ an orthogonal representation over $F$. We give criteria to determine when $\varphi$ lifts to the double cover $\operatorname{Spin}(V)$.

\section{Contents}

1. Introduction

2. Preliminaries

3. Lifting cocharacters

4. Palindromy

5. Irreducible representations

6. Reducible representations

7. Dynkin index

8. Tensor products

9. Type $A_{n-1}$

10. Type $C_{n}$

11. Type $D_{n}$

12. Summary for simple $\mathfrak{g}$

13. Periodicity

14. Reduction to algebraically closed fields

Acknowledgments

References

\section{INTRODUCTION}

Let $G$ be a connected reductive group over a field $F$ of characteristic 0 . Let $(\varphi, V)$ be a representation of $G$, which in this paper always means a finite-dimensional $F$-representation of $G$. Suppose that $V$ is orthogonal, i.e., carries a symmetric nondegenerate bilinear form preserved by $\varphi$. Thus $\varphi$ is a morphism from $G$ to $\mathrm{SO}(V)$. Write $\rho: \operatorname{Spin}(V) \rightarrow \mathrm{SO}(V)$ for the usual isogeny ([SV00]). Following [Bou05], we say that $\varphi$ is spinorial when it lifts to $\operatorname{Spin}(V)$, i.e., provided there

Received by the editors October 31, 2018, and, in revised form, February 21, 2020.

2010 Mathematics Subject Classification. Primary 20G15; Secondary 22E46.

Key words and phrases. Reductive groups, orthogonal representations, Dynkin index, lifting criterion, Weyl dimension formula.

This paper comes out of the first author's Ph.D. thesis at IISER Pune, during which he was supported by an Institute Fellowship. Afterwards he was supported by a fellowship from Bhaskaracharya Pratishthan. 
exists a morphism $\hat{\varphi}: G \rightarrow \operatorname{Spin}(V)$ so that $\varphi=\rho \circ \hat{\varphi}$. We call $\varphi$ aspinorial otherwise.

By an argument in Section [14, we may assume that $F$ is algebraically closed, which we do for the rest of this introduction. Let $T$ be a maximal torus of $G$. Write $\pi_{1}(G)$ for the fundamental group of $G$ (the cocharacter group of $T$ modulo the subgroup $Q(T)$ generated by coroots), and $T_{V}$ for a maximal torus of $\mathrm{SO}(V)$ containing $\varphi(T)$. Then $\varphi$ induces a homomorphism $\varphi_{*}: \pi_{1}(G) \rightarrow \pi_{1}(\mathrm{SO}(V)) \cong$ $\mathbb{Z} / 2 \mathbb{Z}$, and $\varphi$ is spinorial iff $\varphi_{*}$ is trivial. If we take a set of cocharacters $\underline{\nu}=$ $\left\{\nu_{1}, \ldots, \nu_{r}\right\}$ whose images generate $\pi_{1}(G)$, then $\varphi$ is spinorial iff each cocharacter $\varphi_{*} \nu_{i}$ of $T_{V}$ lifts to $\operatorname{Spin}(V)$. (See Section 3 .)

Write $\mathfrak{g}$ for the Lie algebra of $G$, and $X^{*}(T)$ for the character group of $T$. Suppose $(\varphi, V)$ is an orthogonal representation of $G$. Write $C$ for the Casimir element associated to the Killing form. Given a cocharacter $\nu$ of $T$, put

$$
|\nu|^{2}=\sum_{\alpha \in R}\langle\alpha, \nu\rangle^{2} \in 2 \mathbb{Z}
$$

We introduce the integer

$$
p(\underline{\nu})=\frac{1}{2} \operatorname{gcd}\left(\left|\nu_{1}\right|^{2}, \ldots,\left|\nu_{r}\right|^{2}\right) .
$$

Theorem 1. Suppose that $\mathfrak{g}$ is simple and let $\varphi$ be an orthogonal representation of $G$. Then $\varphi$ is spinorial iff the integer

$$
p(\underline{\nu}) \cdot \frac{\operatorname{tr}(C, V)}{\operatorname{dim} \mathfrak{g}}
$$

is even.

Alternatively, this can be reformulated in terms of the Dynkin index "dyn $(\varphi)$ " of $\varphi$ and the dual Coxeter number $\check{h}$ of $\mathfrak{g}$. (We recall these integers in Section 7 .)

Corollary 1. Suppose $\mathfrak{g}$ is simple and let $\varphi$ be an orthogonal representation of $G$. Then $\varphi$ is spinorial iff the integer

$$
p(\underline{\nu}) \cdot \frac{\operatorname{dyn}(\varphi)}{2 \check{h}}
$$

is even.

If $\lambda \in X^{*}(T)$ is dominant, write $\varphi_{\lambda}$ for the irreducible representation with highest weight $\lambda$. As $\lambda$ varies, we may regard (10) as an integer-valued polynomial in $\lambda$. We show that the "spinorial weights" form a periodic subset of the highest weight lattice. To be more precise, let $X_{\text {orth }}^{+} \subset X^{*}(T)$ be the set of highest weights of irreducible orthogonal representations.

Theorem 2. There is a $k \in \mathbb{N}$ so that for all $\lambda_{0}, \lambda \in X_{\text {orth }}^{+}$, the representation $\varphi_{\lambda_{0}}$ is spinorial iff $\varphi_{\lambda_{0}+2^{k} \lambda}$ is spinorial.

For any representation $\varphi$, one can form an orthogonal representation $S(\varphi)=$ $\varphi \oplus \varphi^{\vee}$. When $G$ is semisimple, $S(\varphi)$ is always spinorial. For the reductive case we have the following theorem.

Theorem 3. $S\left(\varphi_{\lambda}\right)$ is spinorial iff the integers

$$
\left\langle\lambda, \nu^{z}\right\rangle \cdot \operatorname{dim} V_{\lambda}
$$

are even for all $\nu \in \underline{\nu}$. 
In this formula, $\nu^{z}$ is the $\mathfrak{z}^{-}$-component of $\nu$ corresponding to the decomposition $\mathfrak{g}=\mathfrak{g}^{\prime} \oplus \mathfrak{z}$, where $\mathfrak{z}$ is the center of $\mathfrak{g}$ and $\mathfrak{g}^{\prime}$ is the derived algebra of $\mathfrak{g}$.

This paper is organized as follows. Section 2 establishes general notation and Section 2.4 sets up preliminaries for the spin groups. In Section 3 we give a criterion for spinoriality in terms of the weights of $\varphi$. This approach is along the lines of [PR95] and Bou05.

We advance the theory in Section 4 by employing an algebraic trick involving palindromic Laurent polynomials; this gives a lifting condition in terms of the integers

$$
q_{\varphi}(\nu)=\left.\frac{1}{2} \cdot \frac{d^{2}}{d t^{2}} \Theta_{\varphi}(\nu(t))\right|_{t=1}
$$

for $\nu \in \underline{\nu}$. Here $\Theta_{\varphi}$ denotes the character of $\varphi$.

In Section 5 we compute $q_{\varphi}(\nu)$ for $\varphi$ irreducible, essentially by taking two derivatives of Weyl's Character Formula. As a corollary we show that every nonabelian reductive group has a nontrivial spinorial irreducible representation. Section 6] works out the case of reducible orthogonal representations, in particular we prove Theorems 1 and 3. In Section 7 we explain the connection with the Dynkin index. Spinoriality for tensor products is understood in Section 8

The next four sections apply our theory to groups $G$ with $\mathfrak{g}$ simple. Our goal is to answer the question: For which such $G$ is every orthogonal representation spinorial? Section 9 covers quotients of $\mathrm{SL}_{n}$, Section 10 covers type $C_{n}$, Section 11 covers type $D_{n}$, and Section 12 presents the final answer to the question.

In Section 13 we prove Theorem 2, the periodicity of the spinorial weights. Finally, in Section 14 we reduce to the case of $F$ algebraically closed.

\section{Preliminaries}

2.1. Notation. Throughout this paper $G$ is a connected reductive algebraic group over $F$ with Lie algebra $\mathfrak{g}$. Until the final section, $F$ is algebraically closed. Write $\mathfrak{g}^{\prime}$ for the derived algebra of $\mathfrak{g}$. We write $T$ for a maximal torus of $G$, with Lie algebra $\mathfrak{t}$ and Weyl group $W$. Put $\mathfrak{t}^{\prime}=\mathfrak{t} \cap \mathfrak{g}^{\prime}$. Let $\operatorname{sgn}: W \rightarrow\{ \pm 1\}$ be the usual sign character of $W$. As in Spr98, let $\left(X^{*}, R, X_{*}, R^{\vee}\right)$ be the root datum associated to G.

The groups $X^{*}=X^{*}(T)=\operatorname{Hom}\left(T, \mathbb{G}_{m}\right)$ and $X_{*}=X_{*}(T)=\operatorname{Hom}\left(\mathbb{G}_{m}, T\right)$ are the character and cocharacter lattices of $T$. One has injections $X^{*} \hookrightarrow \mathfrak{t}^{*}$ and $X_{*} \hookrightarrow \mathfrak{t}$ given by differentiation for the former, and $\nu \mapsto d \nu(1)$ for the latter. We will often identify $X^{*}, R, X_{*}$, and $R^{\vee}$ with their images under these injections. Let $Q(T) \subseteq X_{*}(T)$ be the group generated by the coroots of $T$ in $G$. Write $R^{+}$for a set of positive roots of $T$ in $G$, and $\delta \in \mathfrak{t}^{*}$ for the half-sum of these positive roots. Let $w_{0} \in W$ denote the longest Weyl group element.

For $\lambda, \lambda^{\prime} \in X^{*}(T)$, we write $\lambda^{\prime} \prec \lambda$ when $\lambda-\lambda^{\prime}$ is a nonnegative combination of positive roots.

In this paper all representations $V$ of $G$ are finite-dimensional $F$-representations, equivalently morphisms $\varphi: G \rightarrow \operatorname{GL}(V)$ of algebraic groups. For $\mu \in X^{*}(T)$, write $V^{\mu}$ for the $\mu$-eigenspace of $V$, and put $m_{\varphi}(\mu)=\operatorname{dim} V^{\mu}$, the multiplicity of $\mu$ as a weight of $V$.

If $H$ is an algebraic group, write $H^{\circ}$ for the connected component of the identity. We frequently write $\operatorname{diag}\left(t_{1}, t_{2}, \ldots, t_{n}\right)$ for the $n \times n$ matrix with the given elements as entries. 
2.2. Pairings. Write $\langle,\rangle_{T}: X^{*}(T) \times X_{*}(T) \rightarrow \mathbb{Z}$ for the pairing

$$
\langle\mu, \nu\rangle_{T}=n \Leftrightarrow \mu(\nu(t))=t^{n}
$$

for $t \in F^{\times}$, and $\langle,\rangle_{\mathfrak{t}}: \mathfrak{t}^{*} \times \mathfrak{t}$ for the natural pairing. Note that for $\mu \in X^{*}(T)$ and $\nu \in X_{*}(T)$, we have

$$
\langle d \mu, d \nu(1)\rangle_{\mathfrak{t}}=\langle\mu, \nu\rangle_{T}
$$

So we may drop the subscripts and simply write " $\langle\mu, \nu\rangle$ ".

Write (, ) for the Killing form of $\mathfrak{g}$ restricted to $\mathfrak{t}$; it may be computed by

$$
(x, y)=\sum_{\alpha \in R} \alpha(x) \alpha(y)
$$

for $x, y \in \mathfrak{t}$. Also set $|x|^{2}=(x, x)$. In particular, for $\nu \in X_{*}(T)$ we have $|\nu|^{2}=$ $\sum_{\alpha \in R}\langle\alpha, \nu\rangle^{2}$. The Killing form restricted further to $\mathfrak{t}^{\prime}$ induces an isomorphism $\sigma:\left(\mathfrak{t}^{\prime}\right)^{*} \cong \mathfrak{t}^{\prime}$. We use the same notation " $($,$) " to denote the inverse form on \left(\mathfrak{t}^{\prime}\right)^{*}$ defined for $\mu_{1}, \mu_{2} \in \mathfrak{t}^{\prime}$ by

$$
\left(\mu_{1}, \mu_{2}\right)=\left(\sigma\left(\mu_{1}\right), \sigma\left(\mu_{2}\right)\right) .
$$

In Bou02 this form on $\left(\mathfrak{t}^{\prime}\right)^{*}$ is called the "canonical bilinear form" $\Phi_{R}$. Write $|y|^{2}=(y, y)$ for $y \in\left(\mathfrak{t}^{\prime}\right)^{*}$.

Let $\pi_{1}(G)=X_{*}(T) / Q(T)$. As in the introduction, fix a set $\underline{\nu}=\left\{\nu_{1}, \ldots, \nu_{r}\right\}$ of cocharacters whose images generate $\pi_{1}(G)$, and put

$$
p(\underline{\nu})=\frac{1}{2} \operatorname{gcd}\left(\left|\nu_{1}\right|^{2}, \ldots,\left|\nu_{r}\right|^{2}\right) \text {. }
$$

Often $\underline{\nu}$ will be a singleton $\left\{\nu_{0}\right\}$, in which case we may simply write

$$
p\left(\nu_{0}\right)=p\left(\left\{\nu_{0}\right\}\right)=\frac{1}{2}\left|\nu_{0}\right|^{2} .
$$

2.3. Orthogonal representations. Let $X^{*}(T)^{+}$be the set of dominant characters, i.e., the $\lambda \in X^{*}(T)$ so that $\left\langle\lambda, \alpha^{\vee}\right\rangle \geq 0$ for all $\alpha \in R^{+}$.

Put

$$
X_{\mathrm{sd}}=\left\{\lambda \in X^{*}(T) \mid w_{0} \lambda=-\lambda\right\}
$$

and

$$
X_{\text {orth }}=\left\{\lambda \in X_{\text {sd }} \mid\left\langle\lambda, 2 \delta^{\vee}\right\rangle \text { is even }\right\},
$$

and use the superscript "+" to denote the dominant members of these sets. According to Bou05, $X_{\mathrm{sd}}^{+}$is the set of highest weights of irreducible self-dual representations, and $X_{\text {orth }}^{+}$is the set of highest weights of irreducible orthogonal representations.

For $\lambda \in X^{*}(T)^{+}$, the quantity

$$
|\lambda+\delta|^{2}-|\delta|^{2}=(\lambda, \lambda+2 \delta)
$$

is equal to $\chi_{\lambda}(C)$, the value of the central character of the irreducible representation $\varphi_{\lambda}$ at the Casimir element $C$. (See [Jr.08].) 
2.4. Tori of spin groups. In this section we recall material about the tori of spin groups. Our reference is Section 6.3 of GW09.

For the even-dimensional case, let $V$ be a vector space with basis $\left(e_{1}, \ldots, e_{n}\right.$, $\left.e_{-n}, \ldots, e_{-1}\right)$. For the odd-dimensional case, use the basis $\left(e_{1}, \ldots, e_{n}, e_{0}\right.$, $\left.e_{-n}, \ldots, e_{-1}\right)$. In either case, give $V$ the symmetric bilinear form $($,$) so that$ $\left(e_{i}, e_{-i}\right)=1$ and $\left(e_{i}, e_{j}\right)=0$ for $j \neq-i$.

Let $C(V)$ be the corresponding Clifford algebra, i.e., the quotient of the tensor algebra of $V$ by the relation

$$
v \otimes w+w \otimes v=(v, w) .
$$

Let $\operatorname{Pin}(V)$ denote the subgroup of the invertible elements of $C(V)$, generated by the unit vectors in $V$. The morphism $\rho: \operatorname{Pin}(V) \rightarrow O(V)$ taking each unit vector to the corresponding reflection of $V$ is a double cover. Then $\operatorname{Spin}(V)=\operatorname{Pin}(V)^{\circ}$ is the inverse image of $\mathrm{SO}(V)$ under $\rho$.

For $1 \leq j \leq n$, let $c_{j}(t)=t e_{j} e_{-j}+t^{-1} e_{-j} e_{j} \in \operatorname{Spin}(V)$. This gives a morphism $c_{j}: \mathbb{G}_{m} \rightarrow \operatorname{Spin}(V)$. Define $c: \mathbb{G}_{m}^{n} \rightarrow \operatorname{Spin}(V)$ by

$$
c\left(t_{1}, \ldots, t_{n}\right)=c_{1}\left(t_{1}\right) \cdots c_{n}\left(t_{n}\right) .
$$

The kernel of $c$ is

$$
\left\{\left(t_{1}, \ldots, t_{n}\right) \mid t_{i}= \pm 1, t_{1} \cdots t_{n}=1\right\}
$$

and the image of $c$ is a maximal torus $\tilde{T}_{V}$ of $\operatorname{Spin}(V)$. The image of $\tilde{T}_{V}$ under $\rho$ is the subgroup of diagonal matrices in $\mathrm{SO}(V)$, relative to the basis of $V$ mentioned above. More precisely, the restriction of $\rho$ to $\tilde{T}_{V}$ may be described by

$$
\rho\left(c\left(t_{1}, \ldots, t_{n}\right)\right)=\left\{\begin{array}{l}
\operatorname{diag}\left(t_{1}^{2}, \ldots, t_{n}^{2}, t_{n}^{-2}, \ldots, t_{1}^{-2}\right), \\
\operatorname{diag}\left(t_{1}^{2}, \ldots, t_{n}^{2}, 1, t_{n}^{-2}, \ldots, t_{1}^{-2}\right),
\end{array}\right.
$$

depending on whether $\operatorname{dim} V=2 n$ or $2 n+1$.

The kernel of $\rho$ is generated by $z=c(-1,1, \ldots, 1)=-1 \in C(V)$. Pick $\sqrt{-1} \in F$, and put $c^{+}=c(\sqrt{-1}, \sqrt{-1}, \ldots, \sqrt{-1})$. Then $\left(c^{+}\right)^{2}=z^{n}$.

We now describe the center $Z$ of $\operatorname{Spin}(V)$.

(1) When $\operatorname{dim} V=2 n+1, Z$ is generated by $z$.

(2) When $\operatorname{dim} V=2 n$, with $n$ odd, $Z$ is cyclic of order 4 , generated by $c^{+}$.

(3) When $\operatorname{dim} V=2 n$, with $n$ even, $Z$ is a Klein 4-group generated by $z$ and $c^{+}$.

Define $\vartheta_{i} \in X^{*}\left(T_{V}\right)$ by

$$
\vartheta_{i}: \operatorname{diag}\left(t_{1}, \ldots, t_{n}, \ldots\right) \mapsto t_{i} .
$$

We identify $X^{*}\left(T_{V}\right)$ with $\mathbb{Z}^{n}$ through the bijection $\sum_{i} a_{i} \vartheta_{i} \leftrightarrow\left(a_{1}, \ldots, a_{n}\right)$, and $X_{*}\left(T_{V}\right)$ with $\mathbb{Z}^{n}$ by $\nu \leftrightarrow\left(b_{1}, \ldots, b_{n}\right)$ when $\nu(t)=\operatorname{diag}\left(t^{b_{1}}, \ldots, t^{b_{n}}, \ldots\right)$.

Let $\Sigma$ be a set of weights formed by taking one representative from each pair $\left\{\vartheta_{i},-\vartheta_{i}\right\}$. Then $\Sigma$ is a $\mathbb{Z}$-basis of $X^{*}\left(T_{V}\right)$. Of course, one choice is $\Sigma_{*}=$ $\left\{\vartheta_{1}, \ldots, \vartheta_{n}\right\}$. Put $\omega_{\Sigma}=\sum_{\omega \in \Sigma} \omega$.

Lemma 1. Let $d$ be a positive even integer, and $\zeta_{d} \in F^{\times}$a primitive dth root of unity. Let $\nu \in X_{*}\left(T_{V}\right)$.

(1) $\nu$ lifts to a cocharacter $\tilde{\nu} \in X_{*}\left(\tilde{T}_{V}\right) \Leftrightarrow\left\langle\omega_{\Sigma}, \nu\right\rangle$ is even.

(2) $\nu\left(\zeta_{d}\right)=1 \Leftrightarrow d \mid\left\langle\vartheta_{i}, \nu\right\rangle$ for all $i$.

(3) Assume the conditions in (1) and (2) above. Then $\tilde{\nu}\left(\zeta_{d}\right)=1 \Leftrightarrow 2 d \mid\left\langle\omega_{\Sigma}, \nu\right\rangle$. 
Proof. For the first statement, note that the image of $X_{*}\left(\tilde{T}_{V}\right)$ in $X_{*}\left(T_{V}\right)$ is exactly $Q\left(T_{V}\right)$. One checks that $\left\langle\omega_{\Sigma}, \nu\right\rangle$ is even iff $\nu \in Q\left(T_{V}\right)$.

For the second statement, just use that

$$
\nu(t)=\operatorname{diag}\left(t^{b_{1}}, \ldots, t^{b_{n}}, \ldots\right),
$$

with $b_{i}=\left\langle\vartheta_{i}, \nu\right\rangle$.

Now consider the third statement for $\Sigma=\Sigma_{*}$. By hypothesis each $b_{i}$ in (2) is even, and $\left\langle\omega_{\Sigma_{*}}, \nu\right\rangle=b_{1}+\cdots+b_{n}$ is even. Then

$$
\tilde{\nu}(t)=c\left(t^{b_{1} / 2}, \ldots, t^{b_{n} / 2}\right),
$$

so

$$
\tilde{\nu}\left(\zeta_{d}\right)=c\left(\zeta_{d}^{\frac{b_{1}}{2}}, \ldots, \zeta_{d}^{\frac{b_{n}}{2}}\right)
$$

Since each $b_{i}$ is even, each $\zeta_{d}^{\frac{b_{i}}{2}}= \pm 1$. Therefore $\tilde{\nu}\left(\zeta_{d}\right)=1$, i.e.,

$$
\zeta_{d}^{\frac{b_{1}+\cdots+b_{n}}{2}}=1
$$

equivalently $2 d$ divides $\left\langle\omega_{\Sigma_{*}}, \nu\right\rangle$, as claimed. Finally, by hypothesis $d$ divides each $\left\langle\vartheta_{i}, \nu\right\rangle$, so that $\left\langle\omega_{\Sigma_{*}}, \nu\right\rangle \equiv\left\langle\omega_{\Sigma}, \nu\right\rangle \bmod 2 d$.

\section{LiFTiNG COCHARACTERS}

We reformulate the lifting problem for an orthogonal representation in terms of its weights. Throughout this section $G$ is a connected reductive group over an algebraically closed field $F$, and $T$ is a maximal torus of $G$.

Recall [Spa66] that for nice topological spaces such as manifolds, if $\rho: \tilde{Y} \rightarrow Y$ is a covering map, then a continuous function $\varphi: X \rightarrow Y$ lifts to $\hat{\varphi}: X \rightarrow \tilde{Y}$ iff $\varphi_{*}\left(\pi_{1}(X)\right) \leq \rho_{*}\left(\pi_{1}(\tilde{Y})\right)$ (with compatibly chosen basepoints on $\left.X, Y, \tilde{Y}\right)$. The purpose of the next proposition is to extend this to the setting of algebraic groups.

Lemma 2. Let $G, H$ be connected reductive groups, with maximal tori $T \leq G$ and $T_{H} \leq H$. Let $\varphi: G \rightarrow H$ be a morphism with $\varphi(T) \leq T_{H}$. The induced map $\varphi_{*}: X_{*}(T) \rightarrow X_{*}\left(T_{H}\right)$ takes $Q(T)$ to $Q\left(T_{H}\right)$.

Proof. Suppose first that $G, H$ are semisimple. Write $\rho_{G}: G_{\mathrm{sc}} \rightarrow G$ for the universal cover, with maximal torus $T_{\mathrm{sc}}$ above $T$. Similarly we have $\rho_{H}: H_{\mathrm{sc}} \rightarrow H$, with maximal torus $T_{H, \mathrm{sc}}$. Put $\Phi=\varphi \circ \rho_{G}: G \rightarrow H$.

Let $\tilde{G}=\left(G_{\mathrm{sc}} \times_{H} H_{\mathrm{sc}}\right)^{\circ}$, with projection maps $\mathrm{pr}_{1}: \tilde{G} \rightarrow G_{\mathrm{sc}}$ and $\operatorname{pr}_{2}: \tilde{G} \rightarrow H_{\mathrm{sc}}$. It is easy to see that $\mathrm{pr}_{1}$ is a central isogeny; since $G_{\mathrm{sc}}$ is simply connected, it is an isomorphism by 2.15 of $\operatorname{Spr} 79$. If we put $\widetilde{\varphi}=\mathrm{pr}_{2} \circ\left(\mathrm{pr}_{1}\right)^{-1}$, then the following diagram commutes:

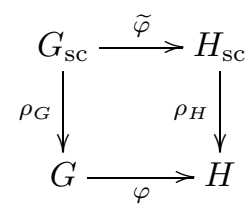


Note that $\varphi$ restricts to a map from $T_{\mathrm{sc}}$ to $T_{H, \mathrm{sc}}$. Applying the covariant functor $T \mapsto X_{*}(T)$ gives the commutative square:

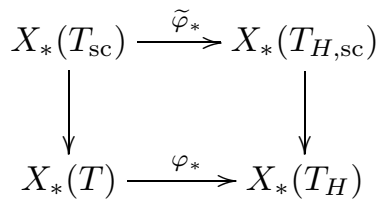

We have $X_{*}\left(T_{\mathrm{sc}}\right)=Q(T)$ and similarly for $T_{H, \mathrm{sc}}$ by simple connectedness. The downward maps, being isogenies, take coroots to coroots and we deduce that $\varphi_{*}$ takes $Q(T)$ to $Q\left(T_{H}\right)$. This conclusion also holds for $G$ and $H$ connected reductive, for one applies the previous argument to the derived groups $G_{\text {der }}$ and $H_{\text {der }}$, recalling that the coroots of $G$ lie in $X_{*}\left(T_{\text {der }}\right)$.

Proposition 1. Let $\rho: \tilde{H} \rightarrow H$ be a central isogeny of connected reductive groups over $F$, and $\varphi: G \rightarrow H$ a morphism. Pick a maximal torus $T_{H} \leq H$ containing $\varphi(T)$, and write $\varphi_{*}: X_{*}(T) \rightarrow X_{*}\left(T_{H}\right)$ for the induced map. Let $\tilde{T}_{H}=\rho^{-1}\left(T_{H}\right) \leq$ $\tilde{H}$, and write $\rho_{*}: X_{*}\left(\tilde{T}_{H}\right) \rightarrow X_{*}\left(T_{H}\right)$ for the induced map. Then there exists a morphism $\hat{\varphi}: G \rightarrow \tilde{H}$ such that $\rho \circ \hat{\varphi}=\varphi$, iff $\operatorname{im} \varphi_{*} \subseteq \operatorname{im} \rho_{*}$. Moreover when this morphism exists, it is unique.

Proof. Let $\tilde{G}=\left(G \times_{H} \tilde{H}\right)^{\circ}$, with projection maps $\rho_{G}: \tilde{G} \rightarrow G$ and $\tilde{\varphi}: \tilde{G} \rightarrow \tilde{H}$. We have the diagram:

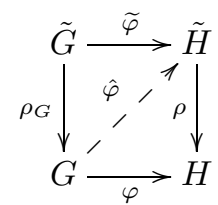

Put $\tilde{T}_{H}=\rho^{-1}\left(T_{H}\right)$ and $\tilde{T}=\rho_{G}^{-1}(T)$. Let us see the equivalence of the following statements:

(1) $\varphi$ lifts to $\hat{\varphi}: G \rightarrow \tilde{H}$.

(2) $\widetilde{\varphi}$ factors through $\rho_{G}$.

(3) $\operatorname{ker} \rho_{G} \leq \operatorname{ker} \widetilde{\varphi}$.

(4) $\operatorname{ker} \rho_{G} \leq\left.\operatorname{ker} \widetilde{\varphi}\right|_{\tilde{T}}$.

(5) $\left.\tilde{\varphi}\right|_{\tilde{T}}$ factors through $T$.

(6) $\left.\varphi\right|_{T}$ lifts to $\tilde{T}_{H}$.

(7) $\varphi_{*}$ lifts in the diagram:

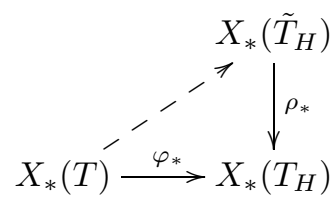

(8) $\operatorname{im} \varphi_{*} \leq \operatorname{im} \rho_{*}$.

For $(1) \Rightarrow(2)$, suppose $\varphi$ lifts to $\hat{\varphi}$. Then

$$
\rho\left(\widetilde{\varphi}(x)^{-1} \cdot \hat{\varphi}\left(\rho_{G}(x)\right)\right)=1 \in H,
$$


so the algebraic map $m: \tilde{G} \rightarrow \tilde{H}$ defined by

$$
m(x)=\widetilde{\varphi}(x)^{-1} \cdot \hat{\varphi}\left(\rho_{G}(x)\right),
$$

takes values in $\operatorname{ker} \rho$. Since $\operatorname{ker} \rho$ is discrete, and $\tilde{G}$ is connected, it must be that $m$ is constant. Thus $m(x)=1 \in \tilde{H}$ for all $x$, i.e., $\widetilde{\varphi}=\hat{\varphi} \circ \rho_{G}$.

For $(2) \Rightarrow(1)$, suppose $\widetilde{\varphi}=\hat{\varphi} \circ \rho_{G}$ for some morphism $\hat{\varphi}$. From the identity $\varphi \circ \rho_{G}=\rho \circ \widetilde{\varphi}$ and the fact that $\rho_{G}$ is surjective we deduce that $\varphi=\rho \circ \hat{\varphi}$.

$(2) \Rightarrow(3)$ is immediate.

The implication $(3) \Rightarrow(2)$ follows from the universal property of $\tilde{G} / \operatorname{ker} \rho_{G}$. (See Section 5.5, page 92 of Spr98.)

Since $\operatorname{ker} \rho_{G} \leq Z(\tilde{G}) \leq \tilde{T}$, we have $(3) \Leftrightarrow(4)$.

The argument for $(4) \Leftrightarrow(5)$ is similar to the argument for $(2) \Leftrightarrow(3)$, and $(5) \Leftrightarrow$ (6) is similar to $(1) \Leftrightarrow(2)$.

To see $(6) \Leftrightarrow(7)$, note that the functors $T \mapsto X_{*}(T)$ and $L \mapsto L \otimes_{\mathbb{Z}} F^{\times}$give an equivalence of categories between $F$-tori and free abelian groups of finite rank.

The equivalence $(7) \Leftrightarrow(8)$ is elementary. Thus (1)-(8) are equivalent.

Finally, suppose that $\hat{\varphi}_{1}$ and $\hat{\varphi}_{2}$ are lifts of $\varphi$. Then $g \mapsto \hat{\varphi}_{1}(g) \hat{\varphi}_{2}(g)^{-1}$ is an algebraic map $G \rightarrow \operatorname{ker} \rho$ taking 1 to 1 . Since $G$ is connected it must be that $\hat{\varphi}_{1}=\hat{\varphi}_{2}$.

Remark 1. This proof did not use the property that $F$ has characteristic zero. In the case of positive characteristic, it is sufficient for $F$ to be separably closed, and then $\hat{\varphi}$ is defined over $F$. Suppose $F$ is an arbitrary field, and the maps $\rho$ and $\varphi$ are defined over $F$. By uniqueness, $\hat{\varphi}$, when it exists, is fixed by the absolute Galois group of $F$ and hence defined over $F$.

By Lemma 2, $\varphi_{*}$ descends to

$$
\varphi_{*}: \pi_{1}(G) \rightarrow \pi_{1}(H) .
$$

Again, since $\rho$ is an isogeny, we have $\rho_{*}\left(Q\left(\tilde{T}_{H}\right)\right)=Q\left(T_{H}\right)$. Therefore a lift $\hat{\varphi}$ in the diagram (4) exists iff $\varphi_{*}$ lifts in the diagram:

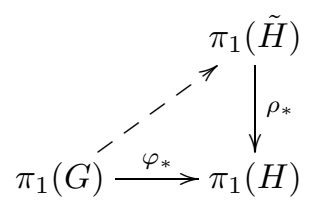

Recall we have fixed a set $\underline{\nu}$ of cocharacters which generates $\pi_{1}(G)$.

Corollary 2. A lift $\hat{\varphi}$ as in the above proposition exists iff $\varphi_{*}(\nu) \in \operatorname{im} \rho_{*}$ for each $\nu \in \underline{\nu}$.

Definition 1. Let $(\varphi, V)$ be a representation of $G$. For $\nu \in X_{*}(T)$, put

$$
L_{\varphi}(\nu)=\sum_{\left\{\mu \in X^{*}(T) \mid\langle\mu, \nu\rangle>0\right\}} m_{\varphi}(\mu)\langle\mu, \nu\rangle \in \mathbb{Z} .
$$

Proposition 2. Let $\varphi: G \rightarrow \mathrm{SO}(V)$ be an orthogonal representation. For $\nu \in$ $X_{*}(T)$, the cocharacter $\varphi_{*}(\nu) \in \operatorname{im} \rho_{*}$ iff $L_{\varphi}(\nu)$ is even. Thus $\varphi$ is spinorial iff the integers $L_{\varphi}(\nu)$ are even for all $\nu \in \underline{\nu}$.

(Compare Exercise 7 in Section 8, Chapter IX of Bou05 and Lemma 3 in [PR95.) 
Proof. We may assume that $\varphi(T) \leq T_{V}$. By Corollary 2 $\varphi$ is spinorial iff $\varphi_{*}(\nu) \in$ $\operatorname{im} \rho_{*}$ for all $\nu \in \underline{\nu}$. By Lemma囵(1), we must check whether the integer $\left\langle\omega_{\Sigma}, \varphi_{*}(\nu)\right\rangle$ is even for a suitable $\Sigma$.

Write $P_{V}=\left\{ \pm \vartheta_{i} \mid 1 \leq i \leq n\right\}$, the weights of $V$ as a $T_{V}$-module. Let

$$
P_{V}^{1}=\left\{\omega \in P_{V} \mid\left\langle\varphi^{*} \omega, \nu\right\rangle \geq 0\right\} .
$$

We may choose $\Sigma \subseteq P_{V}^{1}$ so that $\Sigma$ contains one representative from each pair $\left\{\vartheta_{i},-\vartheta_{i}\right\}$ as in Section 2.4

Then

$$
\begin{aligned}
\left\langle\omega_{\Sigma}, \varphi_{*} \nu\right\rangle & =\sum_{\omega \in \Sigma}\left\langle\varphi^{*} \omega, \nu\right\rangle \\
& =\sum_{\{\mu \mid\langle\mu, \nu\rangle>0\}}\langle\mu, \nu\rangle \cdot \operatorname{dim} V^{\mu} \\
& =L_{\varphi}(\nu) .
\end{aligned}
$$

Thus $\varphi$ lifts iff $L_{\varphi}(\nu)$ is even for all $\nu \in \underline{\nu}$.

Since $\varphi(Q(T)) \subseteq Q\left(T_{V}\right)$ we note the following corollary.

Corollary 3. If $\nu \in Q(T)$, then $L_{\varphi}(\nu)$ is even.

For two representations $\varphi_{1}, \varphi_{2}$, we have

$$
L_{\varphi_{1} \oplus \varphi_{2}}(\nu)=L_{\varphi_{1}}(\nu)+L_{\varphi_{2}}(\nu),
$$

since $m_{\varphi_{1} \oplus \varphi_{2}}(\mu)=m_{\varphi_{1}}(\mu)+m_{\varphi_{2}}(\mu)$.

Corollary 4. The adjoint representation of $G$ on $\mathfrak{g}$ is spinorial iff $\delta \in X^{*}(T)$.

Proof. If $\varphi$ is the adjoint representation, then

$$
\begin{aligned}
L_{\varphi}(\nu) & =\sum_{\{\alpha \in R \mid\langle\alpha, \nu\rangle>0\}}\langle\alpha, \nu\rangle \\
& \equiv \sum_{\alpha \in R^{+}}\langle\alpha, \nu\rangle \bmod 2 \\
& =2\langle\delta, \nu\rangle .
\end{aligned}
$$

The corollary follows since the pairing $X^{*}(T) \times X_{*}(T) \rightarrow \mathbb{Z}$ is perfect.

Remark 2. This is well known; for $G$ a compact connected Lie group, see Section 5.56 of Ada69].

Example 1. Let $G=\mathrm{PGL}_{2}$, with diagonal maximal torus $T$. Then $\pi_{1}(G)$ is generated by $\nu_{0}(t)=\operatorname{diag}(t, 1) \bmod$ center. Let $\alpha$ be the positive root defined by $\alpha(\operatorname{diag}(a, b))=a b^{-1}$, and let $\varphi_{j}$ be the representation of $\mathrm{PGL}_{2}$ with highest weight $j \alpha$. Then

$$
\begin{aligned}
L_{\varphi_{j}}\left(\nu_{0}\right) & =\left\langle\alpha, \nu_{0}\right\rangle+\cdots+\left\langle j \alpha, \nu_{0}\right\rangle \\
& =1+\cdots+j .
\end{aligned}
$$

Therefore $\varphi_{j}$ is spinorial iff $j \equiv 0,3 \bmod 4$. 


\section{Palindromy}

This section is the cornerstone of our paper. The difficulty with determining the parity of $L_{\varphi}(\nu)$ is in somehow getting ahold of "half" of the weights of $V$, one for each positive/negative pair. This amounts to knowledge of the polynomial part of a certain palindromic Laurent polynomial, and this we accomplish with a derivative trick.

Definition 2. For $(\varphi, V)$ a representation of $G$ and $\nu \in X_{*}(T)$, consider the function $Q_{(\varphi, \nu)}: F^{\times} \rightarrow F$ defined by

$$
\begin{aligned}
Q_{(\varphi, \nu)}(t) & =\Theta_{\varphi}(\nu(t)) \\
& =\operatorname{tr}(\varphi(\nu(t))) .
\end{aligned}
$$

If $\varphi$ is understood we may simply write " $Q_{\nu}(t)$ ". For $\gamma \in T$, we have

$$
\Theta_{\varphi}(\gamma)=\sum_{\mu \in X^{*}} m_{\varphi}(\mu) \mu(\gamma)
$$

so in particular

$$
Q_{\nu}(t)=\sum_{\mu \in X^{*}} m_{\varphi}(\mu) t^{\langle\mu, \nu\rangle} \in \mathbb{Z}\left[t, t^{-1}\right]
$$

We note:

- $Q_{\nu}(1)=\operatorname{dim} V$,

- $Q_{\nu}^{\prime}(1)=\sum_{\mu} m_{\varphi}(\mu)\langle\mu, \nu\rangle$,

- $Q_{\nu}^{\prime \prime}(1)=\sum_{\mu}\left(m_{\varphi}(\mu)\langle\mu, \nu\rangle^{2}-m_{\varphi}(\mu)\langle\mu, \nu\rangle\right)$.

Definition 3. For $(\varphi, V)$ a representation of $G$ and $\nu \in X_{*}(T)$, we set

$$
q_{\varphi}(\nu)=\frac{1}{2} Q_{\nu}^{\prime \prime}(1)
$$

When $\varphi$ is self-dual, $m_{\varphi}(-\mu)=m_{\varphi}(\mu)$ for all $\mu \in X^{*}$, so in this case:

- $Q_{\nu}(t)=Q_{\nu}\left(t^{-1}\right)$, i.e., $Q_{\nu}$ is "palindromic",

- $Q_{\nu}^{\prime}(1)=0$,

- $Q_{\nu}^{\prime \prime}(1)=\sum_{\mu} m_{\varphi}(\mu)\langle\mu, \nu\rangle^{2} \in 2 \mathbb{Z}$.

In particular, $q_{\varphi}(\nu)$ is an integer for all $\nu \in X_{*}$.

Lemma 3. For $\varphi$ self-dual and $\nu_{1}, \nu_{2} \in X_{*}$, we have

$$
q_{\varphi}\left(\nu_{1}+\nu_{2}\right) \equiv q_{\varphi}\left(\nu_{1}\right)+q_{\varphi}\left(\nu_{2}\right) \bmod 2 .
$$

Proof. Breaking the sum over $\mu$ into a sum over nonzero pairs $\{\mu,-\mu\}$ gives

Therefore

$$
\begin{aligned}
q_{\varphi} & =\frac{1}{2} \sum_{\mu \in X^{*}} m_{\varphi}(\mu)\langle\mu, \nu\rangle^{2} \\
& =\sum_{\{\mu,-\mu\}} m_{\varphi}(\mu)\langle\mu, \nu\rangle^{2} \in \mathbb{Z} .
\end{aligned}
$$

$$
\begin{aligned}
q_{\varphi}\left(\nu_{1}+\nu_{2}\right) & =\sum_{\{\mu,-\mu\}} m_{\varphi}(\mu)\left(\left\langle\mu, \nu_{1}\right\rangle^{2}+2\left\langle\mu, \nu_{1}\right\rangle\left\langle\mu, \nu_{2}\right\rangle+\left\langle\mu, \nu_{2}\right\rangle^{2}\right) \\
& \equiv q_{\varphi}\left(\nu_{1}\right)+q_{\varphi}\left(\nu_{2}\right) \bmod 2 .
\end{aligned}
$$


Thus when $\varphi$ is self-dual, the function $q_{\varphi}: X_{*} \rightarrow \mathbb{Z}$ induces a group homomorphism $\bar{q}_{\varphi}: X_{*} \rightarrow \mathbb{Z} / 2 \mathbb{Z}$. Our goal in this section is to show that

$$
\bar{q}_{\varphi}(\nu)=L_{\varphi}(\nu) \bmod 2
$$

when $\varphi$ is orthogonal.

Since $Q_{\nu}$ is palindromic, it may be expressed in the form

$$
Q_{\nu}(t)=H_{\nu}(t)+H_{\nu}\left(t^{-1}\right)
$$

for a unique polynomial $H_{\nu} \in \mathbb{Z}[t]+\frac{1}{2} \mathbb{Z}$. Thus $H_{\nu}$ has integer coefficients, except its constant term may be half-integral. More precisely,

$$
H_{\nu}(t)=\sum_{\langle\mu, \nu\rangle>0} m_{\varphi}(\mu) t^{\langle\mu, \nu\rangle}+\frac{1}{2} \sum_{\langle\mu, \nu\rangle=0} m_{\varphi}(\mu) .
$$

What we want, at least $\bmod 2$, is the integer

$$
H_{\nu}^{\prime}(1)=\sum_{\langle\mu, \nu\rangle>0} m_{\varphi}(\mu)\langle\mu, \nu\rangle=L_{\varphi}(\nu) .
$$

By calculus we compute

$$
Q_{\nu}^{\prime \prime}(1)=2\left(H_{\nu}^{\prime}(1)+H_{\nu}^{\prime \prime}(1)\right)
$$

But $H_{\nu}^{\prime \prime}(1)$ is even! This gives the following crucial result.

Proposition 3. If $\varphi$ is self-dual, then

$$
L_{\varphi}(\nu) \equiv q_{\varphi}(\nu) \quad \bmod 2 .
$$

Corollary 5. Let $\varphi$ be an orthogonal representation of $G$. Then $\varphi$ is spinorial iff $q_{\varphi}(\nu)$ is even for every $\nu \in \underline{\nu}$.

Proof. This follows from Corollary 2, Proposition 2, and the above equation.

\section{IRREDUCIBLE REPRESENTATIONS}

In this section we compute $q_{\varphi}(\nu)$ when $\varphi$ is irreducible (not necessarily selfdual). Our method follows the proof of Weyl's Character Formula in GW09. For $\lambda \in X^{*}(T)^{+}$, write $\left(\varphi_{\lambda}, V_{\lambda}\right)$ for the irreducible representation of $G$ with highest weight $\lambda$. For simplicity, we use the notation $q_{\lambda}, m_{\lambda}(\mu)$, etc. for $q_{\varphi_{\lambda}}, m_{\varphi_{\lambda}}(\mu)$, etc.

5.1. Two derivatives of Weyl's Character Formula. For $\nu \in \mathfrak{t}$, put

$$
d_{\nu}=\prod_{\alpha \in R^{+}}\langle\alpha, \nu\rangle
$$

and for $\mu \in \mathfrak{t}^{*}$, put

$$
d_{\mu}=\prod_{\alpha \in R^{+}}\left\langle\mu, \alpha^{\vee}\right\rangle
$$

Definition 4. Put

$$
\mathfrak{t}_{\text {reg }}=\left\{\nu \in \mathfrak{t} \mid d_{\nu} \neq 0\right\} .
$$

Extend the function $q_{\lambda}: X_{*} \rightarrow \mathbb{Z}$ to the polynomial function $q_{\lambda}: \mathfrak{t} \rightarrow F$ defined by the formula

$$
q_{\lambda}(\nu)=\frac{1}{2} \sum_{\mu \in X^{*}}\langle\mu, \nu\rangle^{2} m_{\lambda}(\mu) .
$$


We let $\mathbb{Z}\left[\mathfrak{t}^{*}\right]$ denote the usual algebra of the monoid $\mathfrak{t}^{*}$ with basis $e^{\mu}$ for $\mu \in \mathfrak{t}^{*}$. It contains the elements

$$
J\left(e^{\mu}\right)=\sum_{w \in W} \operatorname{sgn}(w) e^{w \mu} \text { and } \operatorname{ch}\left(V_{\lambda}\right)=\sum_{\mu \in X^{*}} m_{\lambda}(\mu) e^{\mu} .
$$

Recall the Weyl Character Formula (Prop. 5.10 in [Jan03):

$$
\operatorname{ch}\left(V_{\lambda}\right) J\left(e^{\delta}\right)=J\left(e^{\lambda+\delta}\right) .
$$

Write $\varepsilon: \mathbb{Z}\left[\mathfrak{t}^{*}\right] \rightarrow \mathbb{Z}$ for the $\mathbb{Z}$-linear map so that $\varepsilon\left(e^{\mu}\right)=1$ for all $\mu \in \mathfrak{t}^{*}$ (i.e., the augmentation); it is a ring homomorphism. Given $\nu \in \mathfrak{t}$, write $\frac{\partial}{\partial \nu}: \mathbb{Z}\left[\mathfrak{t}^{*}\right] \rightarrow \mathbb{Z}\left[\mathfrak{t}^{*}\right]$ for the $\mathbb{Z}$-linear map so that $\frac{\partial}{\partial \nu}\left(e^{\mu}\right)=\langle\mu, \nu\rangle e^{\mu}$; it is a $\mathbb{Z}$-derivation. Note that $\varepsilon\left(\operatorname{ch}\left(V_{\lambda}\right)\right)=\operatorname{dim} V_{\lambda}$, and

$$
\left(\varepsilon \circ \frac{\partial^{2}}{\partial \nu^{2}}\right) \operatorname{ch}\left(V_{\lambda}\right)=Q_{\nu}^{\prime \prime}(1)
$$

Proposition 4. For $\nu \in \mathfrak{t}_{\text {reg, }}$, we have

$$
q_{\lambda}(\nu)=\frac{\sum_{w \in W} \operatorname{sgn}(w)\langle w(\lambda+\delta), \nu\rangle^{N+2}}{(N+2) ! d_{\nu}}-\frac{1}{48} \operatorname{dim} V_{\lambda}|\nu|^{2},
$$

where $N=\left|R^{+}\right|$.

Proof. We apply $\varepsilon \circ \frac{\partial^{N+2}}{\partial \nu^{N+2}}$ to both sides of $J\left(e^{\lambda+\delta}\right)=\operatorname{ch}\left(V_{\lambda}\right) J\left(e^{\delta}\right)$. On the left we have

$$
\left(\varepsilon \circ \frac{\partial^{N+2}}{\partial \nu^{N+2}}\right) J\left(e^{\lambda+\delta}\right)=\sum_{w \in W} \operatorname{sgn}(w)\langle w(\lambda+\delta), \nu\rangle^{N+2} .
$$

The right-hand side requires more preparation. For $\alpha \in R^{+}$, let $r_{\alpha}=e^{\alpha / 2}-e^{-\alpha / 2}$. Then:

- $\varepsilon\left(r_{\alpha}\right)=0$,

- $\varepsilon \circ \frac{\partial}{\partial \nu}\left(r_{\alpha}\right)=\langle\alpha, \nu\rangle$,

- $\frac{\partial^{2}}{\partial \nu^{2}} r_{\alpha}=\frac{1}{4}\langle\alpha, \nu\rangle^{2} r_{\alpha}$,

- $J\left(e^{\delta}\right)=\prod_{\alpha \in R^{+}} r_{\alpha}$.

The last equality is a familiar identity from Bou02. We may now apply the following lemma.

Lemma 4. Let $R$ be a commutative ring, $D: R \rightarrow R$ a derivation, and $\varepsilon: R \rightarrow R^{\prime}$ a ring homomorphism. Suppose that $r_{1}, \ldots, r_{N} \in \operatorname{ker} \varepsilon$. Then:

(1) $\varepsilon\left(D^{n}\left(r_{1} \cdots r_{N}\right)\right)=0$ for $0 \leq n<N$.

(2) $\varepsilon\left(D^{N}\left(r_{1} \cdots r_{N}\right)\right)=N ! \prod_{i=1}^{N} \varepsilon\left(D\left(r_{i}\right)\right)$.

(3) If also $D^{2}\left(r_{i}\right) \in \operatorname{ker} \varepsilon$ for all $i$, then $\varepsilon\left(D^{N+1}\left(r_{1} \cdots r_{N}\right)\right)=0$.

(4) Suppose further that there are $c_{i} \in R$ so that $D^{2}\left(r_{i}\right)=c_{i} r_{i}$. Then:

$$
\varepsilon\left(D^{N+2}\left(r_{1} \cdots r_{N}\right)\right)=\frac{(N+2) !}{6}\left(\prod_{i} \varepsilon\left(D\left(r_{i}\right)\right)\right)\left(\sum_{i} c_{i}\right) .
$$

Proof. This follows from the Leibniz rule for derivations:

$$
D^{n}\left(r_{1} \cdots r_{k}\right)=\sum_{i_{1}+\cdots+i_{k}=n}\left(\begin{array}{c}
n \\
i_{1}, \ldots, i_{k}
\end{array}\right) D^{i_{1}}\left(r_{1}\right) \cdots D^{i_{k}}\left(r_{k}\right) .
$$


Thus in our case,

(1) $\left(\varepsilon \circ \frac{\partial^{n}}{\partial \nu^{n}}\right) J\left(e^{\delta}\right)=0$ for $0 \leq n<N$,

(2) $\left(\varepsilon \circ \frac{\partial^{N}}{\partial \nu^{N}}\right) J\left(e^{\delta}\right)=N ! d_{\nu}$,

(3) $\left(\varepsilon \circ \frac{\partial^{N+1}}{\partial \nu^{N+1}}\right) J\left(e^{\delta}\right)=0$,

(4) $\left(\varepsilon \circ \frac{\partial^{N+2}}{\partial \nu^{N+2}}\right) J\left(e^{\delta}\right)=\frac{(N+2) !}{24} d_{\nu} \sum_{\alpha>0}\langle\alpha, \nu\rangle^{2}$.

Now we are ready to consider

$$
\left(\varepsilon \circ \frac{\partial^{N+2}}{\partial \nu^{N+2}}\right)\left(\operatorname{ch}\left(V_{\lambda}\right) J\left(e^{\delta}\right)\right) .
$$

Applying the Leibniz rule to the above gives

$$
\left(\begin{array}{c}
N+2 \\
2
\end{array}\right) Q_{\nu}^{\prime \prime}(1) N ! d_{\nu}+\operatorname{dim} V_{\lambda} \frac{(N+2) !}{24} d_{\nu} \sum_{\alpha>0}\langle\alpha, \nu\rangle^{2} .
$$

Equating this with (9) yields the identity

$$
\sum_{w \in W} \operatorname{sgn}(w)\langle w(\lambda+\delta), \nu\rangle^{N+2}=(N+2) ! d_{\nu}\left(q_{\lambda}(\nu)+\frac{\operatorname{dim} V_{\lambda}}{24} \sum_{\alpha>0}\langle\alpha, \nu\rangle^{2}\right),
$$

whence the proposition.

5.2. Anti- $W$-invariant polynomials. The expression

$$
\sum_{w \in W} \operatorname{sgn}(w)\langle w(\lambda+\delta), \nu\rangle^{N+2}
$$

in our formula demands simplification. This can be done by applying the theory of anti- $W$-invariant polynomials.

Let $f: \mathfrak{t} \rightarrow F$ be a polynomial function. We say that $f$ is anti- $W$-invariant, provided for all $w \in W$ and $\nu \in \mathfrak{t}$ we have

$$
f(w(\nu))=\operatorname{sgn}(w) f(\nu) .
$$

The polynomial $\nu \mapsto d_{\nu}$ is a homogeneous anti- $W$-invariant polynomial of degree $N$. According to Bou02, page 118, if $f$ is a homogeneous anti- $W$-invariant polynomial of degree $d$, then there exists a homogeneous $W$-invariant polynomial $p: \mathfrak{t} \rightarrow F$ so that $f(\nu)=p(\nu) d_{\nu}$. Necessarily $d=\operatorname{deg} f \geq N$ and $p$ has degree $d-N$. Similarly, if $g: \mathfrak{t}^{*} \rightarrow F$ is a homogeneous anti- $W$-invariant polynomial, then $g(\mu)=p(\mu) d_{\mu}$ for a $W$-invariant polynomial $p$ on $\mathfrak{t}^{*}$.

In this section we will make use of the famous Weyl dimension formula, which we recall is $\operatorname{dim} V_{\lambda}=d_{\lambda+\delta} / d_{\delta}$.

Definition 5. Let $k$ be a nonnegative integer. Put

$$
F_{k}(\mu, \nu)=\sum_{w \in W} \operatorname{sgn}(w)\langle w(\mu), \nu\rangle^{k}
$$

for $\mu \in \mathfrak{t}^{*}$ and $\nu \in \mathfrak{t}$.

Proposition 5. Let $\mathfrak{g}$ be simple. Then

$$
F_{k}(\mu, \nu)= \begin{cases}0 & \text { if } 0 \leq k<N \text { or } k=N+1, \\ N ! \cdot \frac{d_{\mu} d_{\nu}}{d_{\delta}} & \text { if } k=N, \\ \frac{(N+2) !}{48|\delta|^{2}} \cdot \frac{d_{\mu} d_{\nu}}{d_{\delta}}|\mu|^{2}|\nu|^{2} & \text { if } k=N+2 .\end{cases}
$$


Proof. Each $F_{k}$ may be viewed as a polynomial in two ways: as a function of $\mu$ and as a function of $\nu$. It is either identically 0 , or homogeneous of degree $k$. Both the functions $\mu \mapsto F_{k}(\mu, \nu)$ and $\nu \mapsto F_{k}(\mu, \nu)$ are anti- $W$-invariant. Therefore $F_{k}(\mu, \nu)$ either vanishes, or is the product of $d_{\mu} d_{\nu}$ and a homogeneous $W$-invariant polynomial of degree $k-N$ in both $\nu$ and $\mu$. By degree considerations, $F_{k}$ must vanish for $0 \leq k<N$.

Case $k=N$. Here $F_{N}(\mu, \nu)=c d_{\mu} d_{\nu}$ for some constant $c \in F$, independent of $\mu$ and $\nu$. To determine $c$, we apply $\varepsilon \circ \frac{\partial^{N}}{\partial \nu^{N}}$ to both sides of $J\left(e^{\lambda+\delta}\right)=\operatorname{ch}\left(V_{\lambda}\right) J\left(e^{\delta}\right)$. On the left we have

$$
\left(\varepsilon \circ \frac{\partial^{N}}{\partial \nu^{N}}\right) J\left(e^{\lambda+\delta}\right)=F_{N}(\lambda+\delta, \nu)
$$

On the right we proceed as in the proof of Proposition 4 to obtain $N ! \cdot \operatorname{dim} V_{\lambda} \cdot d_{\nu}$. Therefore

so that $c=\frac{N !}{d_{\delta}}$.

$$
c \cdot d_{\lambda+\delta} d_{\nu}=N ! \cdot \operatorname{dim} V_{\lambda} \cdot d_{\nu}
$$

Case $k=N+1$. Since $\mathfrak{g}$ is simple, both $\mathfrak{t}$ and $\mathfrak{t}^{*}$ are irreducible representations of $W$. If $\operatorname{dim} \mathfrak{t}>1$, there is no 1 -dimensional invariant subspace. When $\operatorname{dim} \mathfrak{t}=1$, $W$ acts by a nontrivial reflection. Therefore there is no $W$-invariant vector, i.e., no $W$-invariant polynomial of degree 1 . Thus in all cases $F_{N+1}$ vanishes.

Case $k=N+2$. Let us write $F_{N+2}(\mu, \nu)=\mathcal{Q}_{\mu}(\nu) d_{\nu}$ with $\mathcal{Q}_{\mu}$ a $W$-invariant quadratic form on $\mathfrak{t}$. The corresponding bilinear form on $\mathfrak{t}$ is $W$-invariant; as $\mathfrak{t}$ is an irreducible $W$-representation, this bilinear form must be a scalar multiple of the Killing form. Thus we may write

$$
F_{N+2}(\mu, \nu)=c_{R} d_{\mu} d_{\nu}|\mu|^{2}|\nu|^{2} ;
$$

it remains to determine $c_{R}$.

Let $\sigma$ be as in Section 2.2. Employing Bou05, Ch. VIII, Section 9, Exercise 7, we obtain the value at $\nu=\sigma(\delta) \in \mathfrak{t}$ :

$$
\begin{aligned}
Q_{\sigma(\delta)}^{\prime \prime}(1) & =\sum_{\mu}\langle\mu, \sigma(\delta)\rangle^{2} m_{\lambda}(\mu) \\
& =\frac{\operatorname{dim} V_{\lambda}}{24} \cdot(\lambda, \lambda+2 \delta) .
\end{aligned}
$$

Substituting this into (10) gives

$$
\begin{aligned}
F_{N+2}(\lambda+\delta, \sigma(\delta)) & =\frac{1}{2} d_{\sigma(\delta)}(N+2) !\left(Q_{\sigma(\delta)}^{\prime \prime}(1)+\frac{\operatorname{dim} V_{\lambda}}{24}|\delta|^{2}\right) \\
& =d_{\sigma(\delta)}(N+2) ! \frac{\operatorname{dim} V_{\lambda}}{48}|\lambda+\delta|^{2} .
\end{aligned}
$$

On the other hand, from (12) we have

$$
\begin{aligned}
F_{N+2}(\lambda+\delta, \sigma(\delta)) & =c_{R} d_{\lambda+\delta} d_{\sigma(\delta)}|\lambda+\delta|^{2}|\delta|^{2} \\
& =c_{R} \operatorname{dim} V_{\lambda} d_{\delta} d_{\sigma(\delta)}|\lambda+\delta|^{2}|\delta|^{2} .
\end{aligned}
$$

We deduce that

$$
c_{R}=\frac{(N+2) !}{48 d_{\delta}|\delta|^{2}} .
$$

The proposition follows from this. 
For the general case, say that $\mathfrak{g}=\mathfrak{g}^{1} \oplus \cdots \oplus \mathfrak{g}^{\ell} \oplus \mathfrak{z}$ with each $\mathfrak{g}^{i}$ simple, and $\mathfrak{z}$ abelian. A Cartan subalgebra $\mathfrak{t} \subset \mathfrak{g}$ is the direct sum of the center $\mathfrak{z}$ and Cartan subalgebras $\mathfrak{t}^{i} \subset \mathfrak{g}^{i}$, and the Weyl group $W=W(\mathfrak{g}, \mathfrak{t})$ is the direct product of the Weyl groups $W^{i}=W^{i}\left(\mathfrak{g}^{i}, \mathfrak{t}^{i}\right)$. Any $\mu \in \mathfrak{t}^{*}$ is equal to $\mu^{z}+\sum_{i} \mu^{i}$ with $\mu^{i} \in\left(\mathfrak{t}^{i}\right)^{*}$ and $\mu^{z} \in \mathfrak{z}^{*}$; similarly for $\nu \in \mathfrak{t}$. Let $N_{i}$ (resp., $N$ ) be the number of positive roots in $\mathfrak{g}^{i}$ (resp., $\left.\mathfrak{g}\right)$.

Proposition 6. Let $\mu \in \mathfrak{t}^{*}$ and $\nu \in \mathfrak{t}$, with notation as above. Then

$$
F_{k}(\mu, \nu)= \begin{cases}0 & \text { if } 0 \leq k<N, \\ N ! \cdot \frac{d_{\mu} d_{\nu}}{d_{\delta}} & \text { if } k=N, \\ (N+1) ! \cdot \frac{d_{\mu} d_{\nu}}{d_{\delta}}\left\langle\mu^{z}, \nu^{z}\right\rangle & \text { if } k=N+1, \\ \frac{(N+2) !}{48} \cdot \frac{d_{\mu} d_{\nu}}{d_{\delta}} \sum_{i} \frac{\left|\mu^{i}\right|^{2}\left|\nu^{i}\right|^{2}}{\left|\delta^{i}\right|^{2}} & \\ +\frac{(N+2) !}{2} \cdot \frac{d_{\mu} d_{\nu}}{d_{\delta}}\left\langle\mu^{z}, \nu^{z}\right\rangle^{2} & \text { if } k=N+2 .\end{cases}
$$

Proof. If $\mathfrak{z}=0$, we have

$$
\begin{aligned}
F_{k}(\mu, \nu) & =\sum_{w \in W} \operatorname{sgn}(w)\left\langle w\left(\mu^{1}+\cdots+\mu^{\ell}\right), \nu^{1}+\cdots+\nu^{\ell}\right\rangle^{k} \\
& =\sum_{w=\left(w_{1}, \ldots, w_{\ell}\right) \in W} \operatorname{sgn}(w)\left(\sum_{i=1}^{\ell}\left\langle w_{i}\left(\mu^{i}\right), \nu^{i}\right\rangle\right)^{k} \\
& =\sum_{w} \operatorname{sgn}(w) \sum_{k_{1}+\cdots+k_{\ell}=k}\left(\begin{array}{c}
k \\
k_{1}, \ldots, k_{\ell}
\end{array}\right) \prod_{i}\left\langle w_{i}\left(\mu^{i}\right), \nu^{i}\right\rangle^{k_{i}} \\
& =\sum_{k_{1}+\cdots+k_{\ell}=k}\left(\begin{array}{c}
k \\
k_{1}, \ldots, k_{\ell}
\end{array}\right) \prod_{i} \sum_{w_{i} \in W^{i}} \operatorname{sgn}\left(w_{i}\right)\left\langle w_{i}\left(\mu^{i}\right), \nu^{i}\right\rangle^{k_{i}} \\
& =\sum_{k_{1}+\cdots+k_{\ell}=k}\left(\begin{array}{c}
k \\
k_{1}, \ldots, k_{\ell}
\end{array}\right) \prod_{i} F_{k_{i}}\left(\mu^{i}, \nu^{i}\right) .
\end{aligned}
$$

The product $\prod_{i} F_{k_{i}}\left(\mu^{i}, \nu^{i}\right)$ vanishes unless $k_{i} \geq N_{i}$ for all $i$. So $F_{k}(\mu, \nu)$ vanishes for $k<N$.

Now put $k=N+2$. Since $k_{1}+\cdots+k_{\ell}=N+2$, we see by Proposition 5 that this product is only nonzero when some $k_{i}=N_{i}+2$ and the other $k_{i}$ equal $N_{i}$. Therefore

$$
\begin{aligned}
& F_{N+2}(\mu, \nu) \\
& \quad=\sum_{i=1}^{\ell}\left(\begin{array}{c}
N+2 \\
N_{1}, \ldots, N_{i}+2, \ldots, N_{\ell}
\end{array}\right) F_{N_{1}}\left(\mu^{1}, \nu^{1}\right) \cdots F_{N_{i}+2}\left(\mu^{i}, \nu^{i}\right) \cdots F_{N_{\ell}}\left(\mu^{\ell}, \nu^{\ell}\right) \\
& \quad=\frac{(N+2) !}{48} \cdot \frac{d_{\mu} d_{\nu}}{d_{\delta}} \sum_{i=1}^{\ell} \frac{\left|\mu^{i}\right|^{2}\left|\nu^{i}\right|^{2}}{\left|\delta_{i}\right|^{2}} .
\end{aligned}
$$

If $\mathfrak{z} \neq 0$, there is an extra term $\frac{(N+2) !}{2} \cdot \frac{d_{\mu} d_{\nu}}{d_{\delta}}\left\langle\mu^{z}, \nu^{z}\right\rangle^{2}$. The other cases are similar. 


\subsection{Main theorem for $\varphi$ irreducible.}

Proposition 7. Let $\mathfrak{g}$ be simple and $\varphi=\varphi_{\lambda}$ irreducible. Then for all $\nu \in \mathfrak{t}$, we have

$$
q_{\lambda}(\nu)=\frac{\operatorname{dim} V_{\lambda} \cdot \chi_{\lambda}(C)}{\operatorname{dim} \mathfrak{g}} \cdot \frac{|\nu|^{2}}{2} .
$$

Proof. Let $\nu \in \mathfrak{t}_{\mathrm{reg}}$. By Proposition 4 ,

$$
\begin{aligned}
q_{\lambda}(\nu) & =\frac{F_{N+2}(\lambda+\delta, \nu)}{(N+2) ! d_{\nu}}-\frac{1}{48} \operatorname{dim} V_{\lambda}|\nu|^{2} \\
& =\frac{1}{48|\delta|^{2}} \cdot \frac{d_{\lambda+\delta}}{d_{\delta}}|\lambda+\delta|^{2}|\nu|^{2}-\frac{1}{48} \operatorname{dim} V_{\lambda}|\nu|^{2} \\
& =\frac{1}{48|\delta|^{2}} \operatorname{dim} V_{\lambda}|\nu|^{2}\left(|\lambda+\delta|^{2}-|\delta|^{2}\right) .
\end{aligned}
$$

Recall that $\chi_{\lambda}(C)=|\lambda+\delta|^{2}-|\delta|^{2}$. Moreover, by Bou05, Exercise 7, page 256, we have $|\delta|^{2}=\operatorname{dim} \mathfrak{g} / 24$. These substitutions give the proposition for the case $\nu \in \mathfrak{t}_{\text {reg }}$; by continuity it holds for $\nu \in \mathfrak{t}$.

Example 2. Revisiting $\mathrm{PGL}_{2}$ from Example1, one computes $\left|\nu_{0}\right|^{2}=2, \operatorname{dim} V_{j \alpha}=$ $2 j+1$, and $\chi_{j \alpha}=\frac{1}{2}\left(j^{2}+j\right)$, so

$$
q_{j \alpha}\left(\nu_{0}\right)=\frac{j(j+1)(2 j+1)}{6} .
$$

So as before $\varphi_{j \alpha}$ is spinorial iff $j \equiv 0,3 \bmod 4$.

The case of $G$ reductive is similar:

Proposition 8. With notation as before, and $\varphi_{\lambda}$ irreducible, we have

$$
q_{\lambda}(\nu)=\frac{1}{2} \operatorname{dim} V_{\lambda} \cdot \sum_{i} \frac{\left|\nu^{i}\right|^{2} \chi_{\lambda^{i}}\left(C^{i}\right)}{\operatorname{dim} \mathfrak{g}^{i}} .
$$

Proof. For $\mathfrak{z}=0$, we have

$$
\begin{aligned}
q_{\lambda}(\nu) & =\frac{F_{N+2}(\lambda+\delta, \nu)}{(N+2) ! d_{\nu}}-\frac{1}{48} \operatorname{dim} V_{\lambda}|\nu|^{2} \\
& =\frac{1}{48} \cdot \operatorname{dim} V_{\lambda} \sum_{i=1}^{\ell} \frac{\left|\lambda^{i}+\delta^{i}\right|^{2}\left|\nu^{i}\right|^{2}}{\left|\delta^{i}\right|^{2}}-\frac{1}{48} \operatorname{dim} V_{\lambda} \sum_{i}\left|\nu_{i}\right|^{2} \\
& =\frac{1}{48} \operatorname{dim} V_{\lambda} \sum_{i=1}^{l}\left|\nu_{i}\right|^{2}\left(\frac{\left|\lambda^{i}+\delta^{i}\right|^{2}-\left|\delta^{i}\right|^{2}}{\left|\delta^{i}\right|^{2}}\right) .
\end{aligned}
$$

The substitution $\left|\delta^{i}\right|^{2}=\operatorname{dim} \mathfrak{g}^{i} / 24$ gives the proposition in the semisimple case. If $\mathfrak{z} \neq 0$, one must add $\frac{1}{2}\left\langle\lambda, \nu^{z}\right\rangle^{2} \cdot \operatorname{dim} V_{\lambda}$. However for $\varphi_{\lambda}$ irreducible orthogonal, necessarily $\lambda$ annihilates the center.

Corollary 6. An irreducible orthogonal representation $\varphi_{\lambda}$ of $G$ is spinorial iff

$$
\frac{1}{2} \operatorname{dim} V_{\lambda} \sum_{i} \frac{\left|\nu^{i}\right|^{2} \chi_{\lambda^{i}}\left(C^{i}\right)}{\operatorname{dim} \mathfrak{g}^{i}}
$$

is even for all cocharacters $\nu \in \underline{\nu}$.

Proof. This follows from Proposition 8 and Corollary 5 
Example 3. For $G=\mathrm{SO}_{4}$, the Lie algebra $\mathfrak{g}$ is not simple. Here, $X^{*}(T)=X_{\mathrm{sd}}=$ $X_{\text {orth }}$, where $T$ is the diagonal torus of $G$.

We may identify $\mathrm{Spin}_{4} \rightarrow \mathrm{SO}_{4}$ with the cover $\mathrm{SL}_{2} \times \mathrm{SL}_{2} \rightarrow \mathrm{SO}_{4}$ as in Exercise 7.16 of [FH91]. In particular, we may identify $\mathfrak{g}$ with the Lie algebra of $\mathfrak{s l}_{2} \times \mathfrak{s l}_{2}$, and $\mathfrak{t}$ with pairs of diagonal matrices in $\mathfrak{s l}_{2} \times \mathfrak{s l}_{2}$. The irreducible representations of $\mathrm{SL}_{2} \times \mathrm{SL}_{2}$ are the external tensor products $V_{a, b}=\operatorname{Sym}^{a} V_{0} \otimes \mathrm{Sym}^{b} V_{0}$, where $V_{0}$ is the standard 2-dimensional representation of $\mathrm{SL}_{2}$. Here $a, b$ are nonnegative integers; the representation $V_{a, b}$ descends to a representation $\varphi_{a, b}$ of $G$ when $a \equiv b$ $\bmod 2$.

Let $\nu_{s}=\operatorname{diag}(s,-s)$; then $\nu_{s, t}=\left(\nu_{s}, \nu_{t}\right) \in \mathfrak{t}$ corresponds to a cocharacter of $T$ iff either $s, t \in \mathbb{Z}$, or $2 s$ and $2 t$ are both odd integers. Proposition 8 gives

$$
\begin{aligned}
q_{\varphi_{a, b}}\left(\nu_{s, t}\right) & =\frac{1}{2}(a+1)(b+1)\left(\frac{4 s^{2} \cdot \frac{1}{4} a(a+2)}{3}+\frac{4 t^{2} \cdot \frac{1}{4} b(b+2)}{3}\right) \\
& =s^{2}(b+1)\left(\begin{array}{c}
a+2 \\
3
\end{array}\right)+t^{2}(a+1)\left(\begin{array}{c}
b+2 \\
3
\end{array}\right) .
\end{aligned}
$$

Since $\pi_{1}(G)$ is generated by $\nu_{\frac{1}{2}, \frac{1}{2}}$, we deduce that $V_{a, b}$ is spinorial iff

$$
(b+1)\left(\begin{array}{c}
a+2 \\
3
\end{array}\right)+(a+1)\left(\begin{array}{c}
b+2 \\
3
\end{array}\right),
$$

which is always a multiple of 4 , is divisible by 8 .

By the following, spinoriality for irreducible orthogonal representations of connected reductive groups reduces to the semisimple case.

Proposition 9. Let $G$ be a connected reductive group and $\varphi: G \rightarrow \mathrm{SO}(V)$ an irreducible orthogonal representation. Then $\varphi$ factors through the quotient $p: G \rightarrow$ $G / Z(G)^{\circ}$, so that $\varphi=\varphi^{\prime} \circ p$ with $\varphi^{\prime}: G / Z(G)^{\circ} \rightarrow \operatorname{SO}(V)$. Moreover $\varphi$ is spinorial iff $\varphi^{\prime}$ is spinorial.

Proof. By Schur's Lemma, $\varphi(Z(G))$ is a subgroup of the scalars in $\mathrm{SO}(V)$, namely $\left\{ \pm \operatorname{id}_{V}\right\}$. Therefore $\varphi\left(Z(G)^{\circ}\right)$ is trivial. This gives the first part, and the second part is similar.

5.4. Existence of spinorial representations. We continue with $G$ connected reductive. Let $a$ be a positive multiple of 4 and $\lambda=a \delta$. Consider the irreducible representation $\left(\varphi_{\lambda}, V_{\lambda}\right)$. It is easy to see that $V_{\lambda}$ is orthogonal, and $\operatorname{dim} V_{\lambda}=$ $(a+1)^{N}$. Therefore for $\nu \in X_{*}(T)$, we have by Corollary 6 .

$$
\begin{aligned}
q_{\lambda}(\nu) & =\frac{1}{2}(a+1)^{N} \sum_{i} \frac{\left|\nu^{i}\right|^{2}\left(\lambda_{i}, \lambda_{i}+2 \delta_{i}\right)}{\operatorname{dim} \mathfrak{g}^{i}} \\
& =\frac{1}{24}(a+1)^{N} a(a+2) \frac{|\nu|^{2}}{2},
\end{aligned}
$$

since $\left(\delta_{i}, \delta_{i}\right)=\frac{\operatorname{dim} \mathfrak{g}_{i}}{24}$ for each $i$.

Therefore $\varphi_{\lambda}$ is spinorial iff the quantity

$$
\frac{p(\underline{\nu})}{24}(a+1)^{N} \cdot a(a+2)
$$

is even. From this we deduce:

(1) The representation $V_{\lambda}$ is spinorial when $a \equiv 0 \bmod 8$.

(2) If $p(\underline{\nu})$ is even, then $V_{\lambda}$ is spinorial. 
In particular, we have the following corollary.

Corollary 7. A nonabelian connected reductive group has a nontrivial irreducible spinorial representation.

Proof. By the above, one may take $\lambda=8 \delta$.

\section{REDUCIBLE REPRESENTATIONS}

In this section we treat the case of $\varphi$ orthogonal, but not necessarily irreducible.

6.1. Spinoriality of $\varphi \oplus \varphi^{\vee}$. For a representation $(\varphi, V)$ of a connected reductive group $G$, consider the orthogonal representation $\left(S(\varphi), V \oplus V^{\vee}\right)$ defined as follows. We give $V \oplus V^{\vee}$ the quadratic form

$$
\mathcal{Q}\left(\left(v, v^{*}\right)\right)=\left\langle v^{*}, v\right\rangle
$$

and write $S(\varphi)$ for the representation of $G$ on $V \oplus V^{\vee}$ given by

$$
{ }^{g}\left(v, v^{*}\right)=\left(\varphi(g) v, \varphi^{\vee}(g) v^{*}\right) .
$$

For $\nu \in X_{*}(T), \mu \in X^{*}(T)$, and $t \in F, \nu(t)$ acts on $V^{\mu}$ by the scalar $t^{\langle\mu, \nu\rangle}$. Therefore we have

$$
\operatorname{det} \varphi(\nu(t))=t^{s_{\varphi}(\nu)}
$$

where

$$
s_{\varphi}(\nu)=\sum_{\mu \in X^{*}(T)} m_{\varphi}(\mu)\langle\mu, \nu\rangle .
$$

Proposition 10. $L_{S(\varphi)}(\nu) \equiv s_{\varphi}(\nu) \bmod 2$. Therefore $S(\varphi)$ is spinorial iff $s_{\varphi}(\nu)$ is even for all $\nu \in \underline{\nu}$. If $G$ is semisimple, then $S(\varphi)$ is spinorial.

Proof. Since $m_{\varphi \vee}(\mu)=m_{\varphi}(-\mu)$, we have

$$
\begin{aligned}
L_{S(\varphi)}(\nu) & =\sum_{\{\mu \mid\langle\mu, \nu\rangle>0\}}\left(m_{\varphi}(\mu)+m_{\varphi}(-\mu)\right)\langle\mu, \nu\rangle \\
& \equiv \sum_{\{\mu \mid\langle\mu, \nu\rangle>0\}}\left(m_{\varphi}(\mu)-m_{\varphi}(-\mu)\right)\langle\mu, \nu\rangle \bmod 2 \\
& =s_{\varphi}(\nu) .
\end{aligned}
$$

When $G$ is semisimple, the image of $\varphi$ lies in $\operatorname{SL}(V)$, and so $s_{\varphi}(\nu)=0$. Therefore $L_{S(\varphi)}(\nu)$ is even and so $S(\varphi)$ is spinorial in this case.

Now, assume $\varphi=\varphi_{\lambda}$ is irreducible. Let $\nu=\nu^{z}+\nu^{\prime}$ correspond to the decomposition $\mathfrak{t}=\mathfrak{z} \oplus \mathfrak{t}^{\prime}$.

Theorem 4. $S\left(\varphi_{\lambda}\right)$ is spinorial iff the integers

$$
\left\langle\lambda, \nu^{z}\right\rangle \cdot \operatorname{dim} V_{\lambda}
$$

are even for all $\nu \in \underline{\nu}$.

Proof. Differentiating both sides of (13) at $t=1$ gives

$$
s_{\varphi}(\nu)=\operatorname{tr} d \varphi(d \nu(1)),
$$

where $\operatorname{tr}: \mathfrak{t}_{V} \rightarrow F$ is the trace. 
Write $\mathfrak{z}_{V}$ for the center of the Lie algebra of GL( $\left.V\right)$, and $\mathfrak{t}_{V}^{\prime}$ for the Lie algebra of the maximal torus in $\mathrm{SL}(V)$. We have a direct sum decomposition $\mathfrak{t}_{V}=\mathfrak{t}_{V}^{\prime} \oplus \mathfrak{z} V$, and similarly for $\mathfrak{t}$. Let $\operatorname{pr}_{V}: \mathfrak{t}_{V} \rightarrow \mathfrak{z} V$ and $\mathrm{pr}: \mathfrak{t} \rightarrow \mathfrak{z}$ be the projections.

Note that the diagram

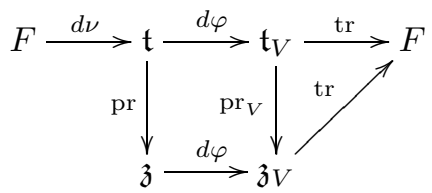

is commutative. Moreover $\operatorname{tr}(d \varphi(z))=d \lambda(z) \cdot \operatorname{dim} V_{\lambda}$ for $z \in \mathfrak{z}$, by Schur's Lemma. It follows that

$$
s_{\varphi}(\nu)=d \lambda\left(\nu^{z}\right) \cdot \operatorname{dim} V_{\lambda},
$$

so the theorem follows from the previous proposition.

Example 4. Let $G=\mathrm{GL}_{2}$. We may parametrize $X^{*}(T)^{+}$with integers $(m, n)$ with $0 \leq n \leq m$ via:

$$
\lambda_{m, n}\left(\begin{array}{cc}
t_{1} & \\
& t_{2}
\end{array}\right)=t_{1}^{m} t_{2}^{n} .
$$

Let $\nu_{0}(t)=\left(\begin{array}{ll}t & 0 \\ 0 & 1\end{array}\right)$, so that $\left(\nu_{0}\right)^{z}=\frac{1}{2}(1,1)$. Then $\operatorname{dim} V_{\lambda_{m, n}}=m-n+1$ and $\left\langle\lambda, \nu_{0}^{z}\right\rangle=$ $\frac{1}{2}(m+n)$, so $s_{\lambda_{m, n}}\left(\nu_{0}\right)=\frac{1}{2}(m+n)(m-n+1)$. From Theorem 4, we deduce that the representation $S\left(\varphi_{\lambda_{m, n}}\right)$ of $\mathrm{GL}_{2}$ is spinorial iff the integer $\frac{1}{2}(m+n)(m-n+1)$ is even.

6.2. General lifting condition. We begin this section by gathering our results to give a general lifting condition for reducible orthogonal representations.

Recall we have $\mathfrak{g}=\mathfrak{g}^{1} \oplus \cdots \oplus \mathfrak{g}^{\ell} \oplus \mathfrak{z}$ with each $\mathfrak{g}^{i}$ simple and $\mathfrak{z}$ abelian. Thus our $\nu \in \mathfrak{t}$ decomposes into $\nu^{z}+\sum_{i} \nu^{i}$ with $\nu^{i} \in \mathfrak{t}^{i}$ and $\nu^{z} \in \mathfrak{z}$.

Proposition 11. If $\varphi$ is an orthogonal representation of $G$, then $\varphi$ is a direct sum of representations of the following type:

- Irreducible orthogonal representations.

- The representations $S(\sigma)$, with $\sigma$ irreducible.

Proof. This follows from Lemma C in Section 3.11 of Sam90].

Theorem 5. Let $\varphi=S(\sigma) \oplus \bigoplus_{j} \varphi_{j}$, with each $\varphi_{j}$ irreducible orthogonal with highest weight $\lambda_{j}$, and $\sigma=\bigoplus_{k} \sigma_{k}$, with each $\sigma_{k}$ irreducible with highest weight $\gamma_{k}$. Then $\varphi$ is spinorial iff for all $\nu \in \underline{\nu}$, the integer

$$
q_{\varphi}(\nu)=\sum_{k}\left\langle\gamma_{k}, \nu^{z}\right\rangle \cdot \operatorname{dim} V_{\gamma_{k}}+\sum_{i} \frac{\left|\nu^{i}\right|^{2}}{2} \sum_{j} \frac{\operatorname{dim} V_{\lambda_{j}} \cdot \chi_{\lambda_{j}^{i}}\left(C^{i}\right)}{\operatorname{dim} \mathfrak{g}^{i}}
$$

is even.

Proof. We have

$$
\begin{aligned}
L_{\varphi}(\nu) & =\sum_{k} L_{S\left(\sigma_{k}\right)}(\nu)+\sum_{j} L_{\varphi_{j}}(\nu) \\
& \equiv \sum_{k} s_{\sigma_{k}}(\nu)+\sum_{j} q_{\varphi_{j}}(\nu) \quad \bmod 2 .
\end{aligned}
$$


The first equality is by (5), and the congruence is by Proposition 10 and Proposition 3. The conclusion then follows from Theorem 4 and Corollary 6 .

Note that when $G$ is semisimple, the sum over $k$ vanishes.

6.3. Case of $\mathfrak{g}$ simple. The situation is much nicer when $\mathfrak{g}$ is simple; let us deduce Theorem 1 as a Corollary of Theorem 5 .

Proof of Theorem 11. By Theorem 5], we have

$$
\begin{aligned}
q_{\varphi}(\nu) & =\frac{|\nu|^{2}}{2} \sum_{j} \frac{\operatorname{dim} V_{\lambda_{j}} \cdot \chi_{\lambda_{j}}(C)}{\operatorname{dim} \mathfrak{g}} \\
& =\frac{|\nu|^{2}}{2} \frac{\operatorname{tr}(C, V)}{\operatorname{dim} \mathfrak{g}} .
\end{aligned}
$$

This must be even for all $\nu \in \underline{\nu}$; equivalently

$$
p(\underline{\nu}) \cdot \frac{\operatorname{tr}(C, V)}{\operatorname{dim} \mathfrak{g}}
$$

must be even.

Corollary 8. Let $\mathfrak{g}$ be simple, and let $\varphi=\varphi_{1} \oplus \varphi_{2}$ with $\varphi_{1}, \varphi_{2}$ orthogonal. Then $\varphi$ is spinorial iff either both $\varphi_{1}, \varphi_{2}$ are spinorial, or both $\varphi_{1}, \varphi_{2}$ are aspinorial.

The following corollary will be useful when varying the isogeny class of $G$.

Corollary 9. Let $\rho: \tilde{G} \rightarrow G$ be a cover, with simple Lie algebra, and let $\underline{\tilde{\nu}}, \underline{\nu}$ be two sets of cocharacters, with $\underline{\tilde{\nu}}$ generating $\pi_{1}(\tilde{G})$ and $\underline{\nu}$ generating $\pi_{1}(G)$. Suppose that $\operatorname{ord}_{2}(p(\underline{\tilde{\nu}}))=\operatorname{ord}_{2}(p(\underline{\nu}))$. Then an orthogonal representation $\varphi$ of $G$ is spinorial iff $\bar{\varphi}=\varphi \circ \rho$ is spinorial.

Proof. This follows since then

$$
\begin{aligned}
\operatorname{ord}_{2}\left(q_{\varphi}\right) & =\operatorname{ord}_{2}(p(\underline{\nu}) \tau(\varphi)) \\
& =\operatorname{ord}_{2}(p(\underline{\tilde{\nu}}) \tau(\bar{\varphi})) \\
& =\operatorname{ord}_{2}\left(q_{\bar{\varphi}}\right) .
\end{aligned}
$$

6.4. A counterexample. The simplicity hypothesis for Corollary 8 is necessary, for example let $G_{1}$ and $G_{2}$ be connected semisimple groups, with orthogonal representations $\left(\varphi_{1}, V_{1}\right)$ and $\left(\varphi_{2}, V_{2}\right)$, respectively. Let $G=G_{1} \times G_{2}$, and write $\Phi_{i}: G_{1} \times G_{2} \rightarrow \operatorname{SO}\left(V_{i}\right)$ for the inflations of $\varphi_{1}, \varphi_{2}$ to $G$ via the two projections. Put $\Phi=\Phi_{1} \oplus \Phi_{2}$. For $\nu_{1}, \nu_{2}$ cocharacters of tori of $G_{1}, G_{2}$, put $\nu=\nu_{1} \times \nu_{2}$. It is easy to see that

$$
L_{\Phi}(\nu)=L_{\varphi_{1}}\left(\nu_{1}\right)+L_{\varphi_{2}}\left(\nu_{2}\right) .
$$

Therefore in this situation,

$$
\begin{aligned}
\Phi \text { is spinorial } & \Leftrightarrow \varphi_{1} \text { and } \varphi_{2} \text { are spinorial } \\
& \Leftrightarrow \Phi_{1} \text { and } \Phi_{2} \text { are spinorial. }
\end{aligned}
$$

For example, if $G=\mathrm{SO}(3) \times \mathrm{SO}(3)$, and $\varphi_{1}, \varphi_{2}$ are aspinorial (e.g., the defining representation of $\mathrm{SO}(3))$, then each of $\Phi_{1}, \Phi_{2}$, and $\Phi_{1} \oplus \Phi_{2}$ is aspinorial. 


\section{DYNKIN INDEX}

Let $\mathfrak{g}$ be a simple Lie algebra with a long root $\alpha$. The quantity

$$
\check{h}=\frac{1}{|\alpha|^{2}}
$$

is called the dual Coxeter number of $G$. (See Section 2 of [Kos76].)

Following Dyn52, we define a bilinear form on $\mathfrak{t}$ by

$$
(x, y)_{d}=2 \check{h} \cdot(x, y)
$$

for $x, y \in \mathfrak{g}$. In other words, we renormalize the Killing form so that $(\alpha, \alpha)_{d}=2$.

Definition 6. Let $\phi: \mathfrak{g}_{1} \rightarrow \mathfrak{g}_{2}$ be a homomorphism of simple Lie algebras. Then there exists an integer $\operatorname{dyn}(\phi)$, called the Dynkin index of $\phi$, so that for $x, y \in \mathfrak{g}$, we have

$$
(\phi(x), \phi(y))_{d}=\operatorname{dyn}(\phi) \cdot(x, y)_{d} .
$$

If $\phi \neq 0$, then $\operatorname{dyn}(\phi) \neq 0$. Also, if $f^{\prime}: \mathfrak{g}_{2} \rightarrow \mathfrak{g}_{3}$ is another homomorphism of simple Lie algebras, then $\operatorname{dyn}\left(f^{\prime} \circ f\right)=\operatorname{dyn}\left(f^{\prime}\right) \operatorname{dyn}(f)$. We refer the reader to Dyn00, page 195, Theorem 2.2, and (2.4).

We assume for the rest of this section that $\mathfrak{s o}_{V}$ is simple, equivalently $\operatorname{dim} V \neq$ $1,2,4$. Note that there are no nontrivial irreducible orthogonal representations of $\mathfrak{g}$ with those degrees. The following is an easy calculation.

Lemma 5. If $\iota_{V}: \mathfrak{s o}_{V} \hookrightarrow \mathfrak{s l}_{V}$ is the standard inclusion, then $\operatorname{dyn}\left(\iota_{V}\right)=2$.

Now let $\varphi: \mathfrak{g} \rightarrow \mathfrak{s l}_{V}$ be a nontrivial orthogonal Lie algebra representation. Then we may write $\varphi=\iota_{V} \circ \varphi^{\prime}$, where $\varphi^{\prime}: \mathfrak{g} \rightarrow \mathfrak{s o}_{V}$. We define $\operatorname{dyn}^{o}(\varphi)=\operatorname{dyn}\left(\varphi^{\prime}\right) \in \mathbb{N}$; thus $\operatorname{dyn}(\varphi)=2 \operatorname{dyn}^{o}(\varphi)$.

Theorem 6. For $\varphi: \mathfrak{g} \rightarrow \mathfrak{s l}_{V}$ a representation, we have

$$
\operatorname{dyn}(\varphi)=2 \check{h} \frac{\operatorname{tr}(C ; V)}{\operatorname{dim} \mathfrak{g}} .
$$

Proof. This is a reformulation of Theorem 2.5 of Dyn00, page 197.

Corollary 10. Let $G$ have simple Lie algebra $\mathfrak{g}$, and let $\varphi$ be an orthogonal representation of $G$. For a cocharacter $\nu$ we have

$$
q_{\varphi}(\nu)=\frac{|\nu|^{2}}{2} \cdot \frac{\operatorname{dyn}^{o}(\varphi)}{\check{h}} .
$$

Therefore $\varphi$ is spinorial iff

is even.

$$
p(\underline{\nu}) \cdot \frac{\operatorname{dyn}^{o}(\varphi)}{\check{h}}
$$

This formula is convenient because for simple $\mathfrak{g}$, the dual Coxeter numbers are tabulated in Section 6 of [Kac90], and Dynkin indices for fundamental representations are found in Table 5 of Dyn52. In the forthcoming examples, we will use these tables without further comment.

\section{Tensor PRODUCts}

In this section, we explain how the spinoriality of a tensor product of two orthogonal representations is related to the spinoriality of the factors. 
8.1. Internal products. Let $G$ be a connected reductive group and $\left(\varphi_{1}, V_{1}\right)$, $\left(\varphi_{2}, V_{2}\right)$ orthogonal representations of $G$. Write $(\varphi, V)=\left(\varphi_{1} \otimes \varphi_{2}, V_{1} \otimes V_{2}\right)$ for the (internal) tensor product representation of $G$.

Proposition 12. For $\nu \in X_{*}(T)$, we have

$$
q_{\varphi}(\nu)=\operatorname{dim} V_{1} \cdot q_{\varphi_{2}}(\nu)+\operatorname{dim} V_{2} \cdot q_{\varphi_{1}}(\nu) .
$$

Proof. For $t \in F^{\times}$, we have

$$
\Theta_{\varphi}(\nu(t))=\Theta_{\varphi_{1}}(\nu(t)) \Theta_{\varphi_{2}}(\nu(t)) .
$$

Therefore

$$
Q_{(\varphi, \nu)}^{\prime \prime}=Q_{\left(\varphi_{1}, \nu\right)} Q_{\left(\varphi_{2}, \nu\right)}^{\prime \prime}+2 Q_{\left(\varphi_{1}, \nu\right)}^{\prime} Q_{\left(\varphi_{2}, \nu\right)}^{\prime}+Q_{\left(\varphi_{1}, \nu\right)}^{\prime \prime} Q_{\left(\varphi_{2}, \nu\right)}
$$

and so

$$
Q_{(\varphi, \nu)}^{\prime \prime}(1)=\operatorname{dim} V_{1} \cdot Q_{\left(\varphi_{2}, \nu\right)}^{\prime \prime}(1)+\operatorname{dim} V_{2} \cdot Q_{\left(\varphi_{1}, \nu\right)}^{\prime \prime}(1) .
$$

The proposition follows.

Corollary 11. If $\varphi_{1}, \varphi_{2}$ are spinorial, then so is $\varphi_{1} \otimes \varphi_{2}$.

8.2. External tensor products. Next, let $\left(\varphi_{1}, V_{1}\right),\left(\varphi_{2}, V_{2}\right)$ be orthogonal representations of connected reductive groups $G_{1}, G_{2}$, respectively. Write $(\varphi, V)=$ $\left(\varphi_{1} \otimes \varphi_{2}, V_{1} \otimes V_{2}\right)$ for the external tensor product representation of $G=G_{1} \times G_{2}$. If $T_{1}, T_{2}$ are maximal tori for $G_{1}, G_{2}$, then $T=T_{1} \times T_{2}$ is a maximal torus of $G$.

As in the previous proposition, we have:

Proposition 13. For $\nu=\left(\nu_{1}, \nu_{2}\right) \in X_{*}(T)=X_{*}\left(T_{1}\right) \oplus X_{*}\left(T_{2}\right)$, we have

$$
q_{\varphi}(\nu)=\operatorname{dim} V_{1} \cdot q_{\varphi_{2}}\left(\nu_{2}\right)+\operatorname{dim} V_{2} \cdot q_{\varphi_{1}}\left(\nu_{1}\right) .
$$

8.3. Positive orthogonal spanning sets. In the examples to come, it will be convenient to have a set of orthogonal dominant weights of $G$ which play the role of fundamental weights, but in $X_{\text {orth }}^{+}$.

Definition 7. Let $S_{o}$ be a set of dominant orthogonal weights. We say that $S_{o}$ is a positive orthogonal spanning set (POSS) for $G$, provided every dominant orthogonal weight can be written as a nonnegative integral combination of $S_{o}$.

The strategy will be to deduce the spinoriality of an arbitrary $\varphi_{\lambda}$ from the spinoriality of the representations $\varphi_{\lambda_{0}}$ with $\lambda_{0} \in S_{0}$.

Lemma 6. Let $G$ be semisimple and $\mu_{0}, \nu_{0}$ dominant weights. Put $\lambda_{0}=\mu_{0}+\nu_{0}$. Suppose that $\Phi=\varphi_{\mu_{0}} \otimes \varphi_{\nu_{0}}$ is spinorial, and that one of the following conditions holds:

(1) $\varphi_{\lambda}$ is spinorial for any dominant orthogonal $\lambda \neq \lambda_{0}$ with $\lambda \prec \lambda_{0}$.

(2) $\varphi_{\lambda}$ is spinorial for any dominant orthogonal $\lambda$ with $|\lambda|<\left|\lambda_{0}\right|$.

Then $\varphi_{\lambda_{0}}$ is spinorial.

Proof. By Proposition 11, $\Phi$ decomposes into a sum of irreducible orthogonal representations $\varphi_{\lambda}$ possibly together with an $S(\sigma)$ summand.

Let us see that each $\varphi_{\lambda}$ is spinorial, for $\lambda \neq \lambda_{0}$. If the first condition holds, this is clear by (Bou05], page 132, Proposition 9 i).

Suppose the second condition holds. Each weight $\lambda$ of $\Phi$ decomposes into $\lambda=$ $\mu_{1}+\nu_{1}$, with $\mu_{1}$ a weight of $\varphi_{\mu_{0}}$ and $\nu_{1}$ a weight of $\varphi_{\nu_{0}}$. Therefore $\mu_{0}-\mu_{1}$ and $\nu_{0}-\nu_{1}$ are positive. Moreover $\varphi_{\lambda_{0}}$ itself occurs with multiplicity one. 
The inner product of a dominant weight with a positive one is nonnegative, thus

$$
\begin{aligned}
|\lambda|^{2} & \leq\left(\lambda, \mu_{0}+\nu_{0}\right) \\
& \leq\left|\mu_{0}\right|^{2}+\left|\nu_{0}\right|^{2}+\left(\mu_{0}, \nu_{1}\right)+\left(\mu_{1}, \nu_{0}\right) \\
& \leq\left|\lambda_{0}\right|^{2} .
\end{aligned}
$$

Moreover by Bou05, page 129, Proposition 5(iii), equality holds iff $\mu_{0}=\mu_{1}$ and $\nu_{0}=\nu_{1}$, i.e., iff $\lambda=\lambda_{0}$. Thus by the second condition, each orthogonal weight $\lambda$ of $\Phi$, except a priori $\lambda_{0}$, has $\varphi_{\lambda}$ spinorial. Recall that $S(\sigma)$ is spinorial by Proposition 10. So by Corollary 8 , it must be that $\varphi_{\lambda_{0}}$ is spinorial.

Proposition 14. Let $\mathfrak{g}$ be simple and suppose $S_{o}$ is a POSS for $G$. If $\varphi_{\lambda}$ is spinorial for each $\lambda \in S_{o}$, then all orthogonal representations of $G$ are spinorial.

Proof. By Proposition 10 and Corollary 8 we reduce to the case of irreducible orthogonal $\varphi_{\lambda_{0}}$. We prove the proposition by induction on $\left|\lambda_{0}\right|$.

If $\lambda_{0} \in S_{o}$, then $\varphi_{\lambda_{0}}$ is spinorial. Otherwise $\lambda_{0}=\mu_{0}+\nu_{0}$ with $\nu_{0} \in S_{o}$ and $\mu_{0}$ dominant orthogonal. Since

$$
\left|\lambda_{0}\right|^{2}=\left|\mu_{0}\right|^{2}+2\left(\mu_{0}, \nu_{0}\right)+\left|\nu_{0}\right|^{2}>\left|\mu_{0}\right|^{2},
$$

we can say that $\varphi_{\mu_{0}}$ is spinorial. Put $\Phi=\varphi_{\mu_{0}} \otimes \varphi_{\nu_{0}}$.

By Corollary 11] $\Phi$ is a spinorial orthogonal representation of $G$. Therefore $\varphi_{\lambda_{0}}$ is spinorial, by Lemma 6 .

\section{TYPE $A_{n-1}$}

For the next few sections, we will pursue the question: For which groups $G$, with $\mathfrak{g}$ simple, is every orthogonal representation spinorial? This section treats the quotients of $\mathrm{SL}_{n}$.

9.1. Preliminaries for type $A_{n-1}$. Let $n$ be an even positive integer. The center of $\mathrm{SL}_{n}$ is cyclic of order $n$, and can be identified with the group $\mu_{n}$ of $n$th roots of unity in $F^{\times}$. Let $T_{1}$ be the diagonal torus of $\mathrm{SL}_{n}$. Let $\vartheta_{i} \in X^{*}\left(T_{1}\right)$ be the character of $T_{1}$ given by taking the $i$ th diagonal entry. The roots of $T_{1}$ are of the form $\vartheta_{i}-\vartheta_{j}$ for $i \neq j$.

Let $d$ be a divisor of $n$, and $\mu_{d}$ the subgroup of $\mu_{n}$ of order $d$. In this section we consider the spinoriality of orthogonal representations of $G_{d}=\mathrm{SL}_{n} / \mu_{d}$. The maximal torus $T_{d}<G_{d}$ is the image of $T_{1}$ under this quotient.

Recall that generally $X_{*}\left(T_{d}\right)$ injects into $\mathfrak{t}$ by $\nu \mapsto d \nu(1)$. When $T_{n}$ is the diagonal torus of $\mathrm{PGL}_{n}$, the injection $X_{*}\left(T_{n}\right) \hookrightarrow \mathfrak{t}$ can be identified with the natural injection

$$
\frac{\mathbb{Z}^{n}}{\mathbb{Z}(1,1, \ldots, 1)} \hookrightarrow \frac{F^{n}}{F(1,1, \ldots, 1)}
$$

In these terms, each coroot lattice $Q\left(T_{d}\right)=X_{*}\left(T_{1}\right)$ is given by

$$
\left\{\left(x_{1}, \ldots, x_{n}\right) \in \frac{\mathbb{Z}^{n}}{\mathbb{Z}(1,1, \ldots, 1)} \mid \sum_{i=1}^{n} x_{i} \equiv 0 \quad \bmod n\right\},
$$

and the subgroup $X_{*}\left(T_{d}\right) \leq X_{*}\left(T_{n}\right)$ is equal to

$$
\left\{\left(x_{1}, \ldots, x_{n}\right) \in \frac{\mathbb{Z}^{n}}{\mathbb{Z}(1,1, \ldots, 1)} \mid \sum_{i=1}^{n} x_{i} \equiv 0 \quad \bmod \frac{n}{d}\right\} .
$$


Let $\nu_{d} \in X_{*}\left(T_{d}\right)$ be the cocharacter parametrized by $\left(\frac{n}{d}, 0, \ldots, 0\right)$ in these terms. Then $\nu_{d}$ generates $\pi_{1}\left(G_{d}\right)$, which is therefore cyclic of order $d$. Of course, when $d$ is odd, every orthogonal representation of $G_{d}$ is spinorial. Let us henceforth take $d$ even. We compute

$$
p\left(\nu_{d}\right)=\left(\frac{n}{d}\right)^{2}(n-1) .
$$

Thus by Theorem 1 we deduce the following proposition.

Proposition 15. Let $\varphi_{\lambda}$ be an irreducible orthogonal representation of $G_{d}$. Then $\varphi_{\lambda}$ is spinorial iff

$$
\left(\frac{n}{d}\right)^{2} \operatorname{dim} V_{\lambda} \cdot \chi_{\lambda}(C)
$$

is even.

(We regard a rational number as even if, when written in lowest terms, its numerator is even.)

Example 5. Let $\varphi_{\lambda}$ be an irreducible orthogonal representation of $\mathrm{GL}_{n}$. By Proposition 9, it descends to an orthogonal representation $\bar{\varphi}_{\lambda}$ of $G_{n}=\mathrm{PGL}_{n}$, and $\varphi_{\lambda}$ is spinorial iff $\bar{\varphi}_{\lambda}$ is. By the above, we deduce that $\varphi_{\lambda}$ is spinorial iff $\operatorname{dim} V_{\lambda} \cdot \chi_{\lambda}(C)$ is even.

Proposition [15] does not by itself answer the question at the beginning of this section, and the groups $G_{d}$ are somewhat awkward to compute with directly. So instead we ask, which morphisms from $\mathrm{SL}_{n}$ to $\operatorname{Spin}(V)$ descend to $G_{d}$ ?

9.2. Descent method. Consider the following approach to determining the spinoriality of an orthogonal $\varphi: G / C \rightarrow \mathrm{SO}(V)$, where $G$ is a simply connected and $C$ is central. Write $\hat{\varphi}: G \rightarrow \operatorname{Spin}(V)$ for the lift of $\varphi$. Then $\varphi$ is spinorial iff $C \leq \operatorname{ker} \hat{\varphi}$. In this subsection we pursue this method for certain $C$; this approach will tremendously simplify the theory for the groups $G / C=G_{d}$ of type $A_{n-1}$.

Resetting notation, let $\hat{\varphi}: G \rightarrow \operatorname{Spin}(V)$ be a morphism, with $G$ connected semisimple. Put $\varphi=\rho \circ \hat{\varphi}: G \rightarrow \mathrm{SO}(V)$, and suppose that $\varphi$ is irreducible. Let $C \leq Z(G)$, and suppose that $C \leq \operatorname{ker} \varphi$. Then $\varphi$ descends to an orthogonal representation $\bar{\varphi}$ of $G / C$, which is spinorial iff $C \leq \operatorname{ker} \hat{\varphi}$.

Let $d$ be a positive even integer, and $\zeta_{d} \in F^{\times}$a primitive $d$ th root of unity. If $\nu \in X_{*}(T)$ with $\nu\left(\zeta_{d}\right) \in C$, then for all weights $\mu$ of $\varphi$, we have $d \mid\langle\mu, \nu\rangle$.

Proposition 16. Let $\nu: \mathbb{G}_{m} \rightarrow T$ be a cocharacter, so that $C$ is generated by $\nu\left(\zeta_{d}\right)$. The following are equivalent:

(1) $\bar{\varphi}$ is spinorial.

(2) $\hat{\varphi}\left(\nu\left(\zeta_{d}\right)\right)=1$.

(3) $2 d$ divides $L_{\varphi}(\nu)$.

Proof. Choose $\Sigma$ as in the proof of Proposition 2, Note that $\hat{\varphi}_{*} \nu \in X_{*}\left(\tilde{T}_{V}\right)$ is a lift of $\varphi_{*} \nu \in X_{*}\left(T_{V}\right)$. By Lemma 1 we have $\hat{\varphi}\left(\nu\left(\zeta_{d}\right)\right)=1$ iff

$$
2 d \mid\left\langle\omega_{\Sigma}, \varphi_{*} \nu\right\rangle
$$

which, as in the proof of Proposition 2, is equal to $L_{\varphi}(\nu)$. 
9.3. Application to $\mathrm{SL}_{n}$. We return to $G=\mathrm{SL}_{n}$, with $n$ even. For $1 \leq i \leq n$, put $\varpi_{i}=\vartheta_{1}+\cdots+\vartheta_{i}$. Let $\nu_{0} \in X_{*}(T)$ be the cocharacter defined by

$$
\nu_{0}(t)=\operatorname{diag}\left(t, t, \ldots, t, t^{1-n}\right) .
$$

Let $d$ be an even divisor of $n$. Then $\nu_{0}\left(\zeta_{d}\right)$ generates $\mu_{d}<G$, so by Proposition 16. the representation $\left(\bar{\varphi}_{\lambda}, V\right)$ of $G_{d}$ is spinorial iff $2 d$ divides $L_{\varphi}\left(\nu_{0}\right)$.

Proposition 17. The adjoint representation of $G_{d}$ is spinorial iff $\frac{n}{d}$ is even.

Proof. For $\varphi=$ ad, we have

$$
\begin{aligned}
L_{\mathrm{ad}}\left(\nu_{0}\right) & =\sum_{\alpha \in R:\left\langle\alpha, \nu_{0}\right\rangle>0}\left\langle\alpha, \nu_{0}\right\rangle \\
& =\sum_{i=1}^{n-1}\left\langle\vartheta_{i}-\vartheta_{n}, \nu_{0}\right\rangle \\
& =n(n-1) .
\end{aligned}
$$

This is divisible by $2 d$ iff $\frac{n}{d}$ is even.

9.4. Case where $n / d$ is even. For all $q \in Q(T)$, the quantity $\left\langle q, \nu_{0}\right\rangle$ is divisible by $n$. Since all weights of $V_{\lambda}$ are congruent $\bmod Q(T)$, we deduce that

$$
L_{\varphi_{\lambda}}\left(\nu_{0}\right) \equiv\left\langle\lambda, \nu_{0}\right\rangle \cdot\left(\sum_{\mu:\left\langle\mu, \nu_{0}\right\rangle>0} m_{\mu}\right) \bmod n .
$$

Proposition 18. Suppose that $2 d$ divides $n$, and an irreducible orthogonal representation $\varphi_{\lambda}$ of $\mathrm{SL}_{n}$ descends to the orthogonal representation $\overline{\varphi_{\lambda}}$ of $G_{d}$.

(1) If $V_{\lambda}$ is odd-dimensional, then $\bar{\varphi}_{\lambda}$ is spinorial.

(2) If $V_{\lambda}$ is even-dimensional, then $\bar{\varphi}_{\lambda}$ is spinorial iff the product $\frac{1}{2} \operatorname{dim} V_{\lambda}$. $\left\langle\lambda, \nu_{0}\right\rangle$ is divisible by $2 d$.

Proof. If $\varphi_{\lambda}$ is orthogonal with odd degree, then the trivial weight must occur in $V_{\lambda}$, which implies that $\lambda \in Q(T)$. From (15), we see that $L_{\varphi}\left(\nu_{0}\right)$ is divisible by $n$, and the first statement follows.

Now suppose $\varphi_{\lambda}$ has even degree. If $\left\langle\lambda, \nu_{0}\right\rangle$ is divisible by $n$, then $\bar{\varphi}_{\lambda}$ is spinorial and the second statement is clear. If $\left\langle\lambda, \nu_{0}\right\rangle$ is not divisible by $n$, then for all $\mu$ occurring in $V_{\lambda}$, it must be that $\left\langle\mu, \nu_{0}\right\rangle \neq 0$. It follows that

$$
\sum_{\mu:\left\langle\mu, \nu_{0}\right\rangle>0} m_{\mu}=\frac{1}{2} \operatorname{dim} V_{\lambda}
$$

and the second statement follows from (15).

Let $\varpi_{i}^{o}=\varpi_{i}+\varpi_{n-i}$ for $1 \leq i<\frac{n}{2}$ and

$$
S_{o}=\left\{\varpi_{i}^{o} \mid 1 \leq i<\frac{n}{2}\right\} \cup\left\{\varpi_{n / 2}\right\} .
$$

It is easy to see that $S_{o}$ is a POSS for $G_{d}$. Note that $\left\langle\varpi_{i}^{o}, \nu_{0}\right\rangle=n$ for $1 \leq i<\frac{n}{2}$, and $\left\langle\varpi_{n / 2}, \nu_{0}\right\rangle=n / 2$.

Proposition 19. Suppose $2 d$ divides n. For each $1 \leq i<\frac{n}{2}$, the representation $V_{\varpi_{i}^{o}}$ of $G_{d}$ is spinorial.

Proof. This follows from Proposition 18. 
The representation $V_{\lambda}$ for $\lambda=\varpi_{n / 2}$ is the exterior power $\bigwedge^{n / 2} V_{0}$, where $V_{0}$ is the standard representation of $\mathrm{SL}_{n}$.

Proposition 20. Let $\lambda=\varpi_{n / 2}$. Then $\bar{\varphi}_{\lambda}$ is aspinorial iff $n$ is a power of 2 and $d=n / 2$.

Proof. From elementary number theory we know that $\operatorname{dim} V_{\lambda}=\left(\begin{array}{c}n \\ n / 2\end{array}\right)$ is even, and divisible by 4 iff $n$ is not a power of 2 . We have

$$
\frac{1}{2} \operatorname{dim} V_{\lambda} \cdot\left\langle\lambda, \nu_{0}\right\rangle=\frac{n}{4}\left(\begin{array}{c}
n \\
n / 2
\end{array}\right) .
$$

This is divisible by $d$, since the binomial coefficient is always even, and $d$ divides $\frac{n}{2}$. Thus (16) is divisible by $2 d$ unless both $\frac{n}{2 d}$ is odd, and $n$ is a power of 2 . In this case it must be that $n$ is a power of 2 and $n=2 d$.

Theorem 7. Suppose that $n / d$ is even. Unless $n=2^{k+1}$ for some $k \geq 1$ and $d=2^{k}$, every orthogonal representation of $G_{d}=\mathrm{SL}_{n} / \mu_{d}$ is spinorial.

Proof. By Proposition 14, it is enough to check that $\bar{\varphi}_{\lambda}$ is spinorial for each $\lambda \in S_{o}$. But we have done this.

Example 6. Although the adjoint representation of $G_{2}=\mathrm{SL}_{4} /\{ \pm 1\}$ is spinorial, the representation $V_{\varpi_{2}}=\bigwedge^{2} F^{4}$ of $G_{2}$ is aspinorial.

Remark 3. What makes the "descent method" work in the case of $G_{d}$ with $n / d$ even is the fortunate fact that $\left\langle q, \nu_{0}\right\rangle$ is divisible by $n$ for $q \in Q(T)$. In other contexts, it is unclear how to compute $L_{\varphi}(\nu) \bmod 2 d$.

9.5. Summary for the groups $G_{d}$. Let $n$ be a positive integer, $d$ a divisor of $n$, and $G_{d}=\mathrm{SL}_{n} / \mu_{d}$.

From the above we have:

- If $d$ is odd, then every orthogonal representation of $G_{d}$ is spinorial.

- If $n$ is even and $n / d$ is odd, then the adjoint representation of $G_{d}$ is aspinorial.

- If $n$ is a power of 2 and $d=n / 2$, then $\bigwedge^{d} V_{0}$ is an aspinorial representation of $G_{d}$.

- If $n / d$ is even, then every orthogonal representation of $G_{d}$ is spinorial, unless $n$ is a power of 2 and $d=n / 2$.

In particular, every orthogonal representation of $G_{d}$ is spinorial iff $n$ is odd, or $n / d$ is even with $(n, d) \neq\left(2^{k+1}, 2^{k}\right)$.

\section{TYPE $C_{n}$}

Let $J$ be the $2 n \times 2 n$ matrix $\left(\begin{array}{cc}0 & I \\ -I & 0\end{array}\right)$, where $I$ is the $n \times n$ identity matrix. We let

$$
G_{\mathrm{sc}}=\mathrm{Sp}_{2 n}=\left\{g \in \mathrm{GL}_{2 n} \mid g^{t} J g=J\right\} .
$$

Write $T_{\mathrm{sc}}$ for the diagonal torus in $G_{\mathrm{sc}}$. A typical element is

$$
\operatorname{diag}\left(t_{1}, \ldots, t_{n}, t_{1}^{-1}, \ldots, t_{n}^{-1}\right) .
$$

We identify $X_{*}\left(T_{\mathrm{sc}}\right)$ with $\mathbb{Z}^{n}$ by $\left(b_{1}, \ldots, b_{n}\right) \mapsto \nu$, where

$$
\nu(t)=\operatorname{diag}\left(t^{b_{1}}, \ldots, t^{b_{n}}, \ldots\right) .
$$




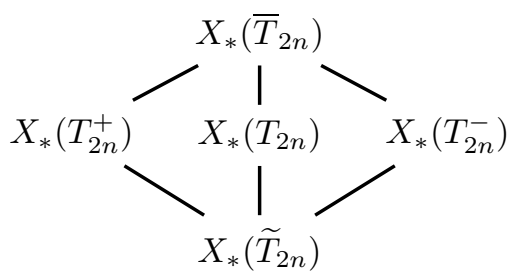

Figure 1. Cocharacter lattice for $D_{2 n}$

Put $G=\mathrm{Sp}_{2 n} /\{ \pm 1\}$, and let $T$ be the image of $T_{\mathrm{sc}}$ under the quotient. Then $X_{*}\left(T_{\mathrm{sc}}\right)$ has index 2 in $X_{*}(T)$; more precisely we may write

$$
X_{*}(T)=X_{*}\left(T_{\mathrm{sc}}\right)+\mathbb{Z} \cdot \nu_{0},
$$

where $\nu_{0}=\frac{1}{2}(1,1, \ldots, 1)$. In particular, $\pi_{1}(G)$ is cyclic of order 2 , generated by $\nu_{0}$.

Remark 4. One way to understand $\nu_{0}$ is through the isomorphism $\operatorname{Sp}_{2 n} /\{ \pm 1\} \cong$ $\mathrm{GSp}_{2 n} / Z$, where $\mathrm{GSp}_{2 n}$ is the general symplectic group defined with $J$, and $Z$ is its center. The cocharacter

$$
t \mapsto \operatorname{diag}(\underbrace{t, t, \ldots, t}_{n \text { times }}, 1,1, \ldots, 1),
$$

of the diagonal torus of $\mathrm{GSp}_{2 n}$, when projected to $T$, is $\nu_{0}$.

We have $p\left(\nu_{0}\right)=\frac{1}{2} n(n+1)$. Every representation $\varphi$ of $G$ is orthogonal. Since $\check{h}=n+1$, we have by Corollary 10 .

$$
\begin{aligned}
q_{\varphi}\left(\nu_{0}\right) & =\frac{\frac{1}{2} n(n+1)}{\check{h}} \operatorname{dyn}^{o}(\varphi) \\
& =\frac{1}{2} n \cdot \operatorname{dyn}^{o}(\varphi) .
\end{aligned}
$$

Proposition 21. Every representation of $\mathrm{Sp}_{2 n} /\{ \pm 1\}$ is spinorial iff $4 \mid n$.

Proof. If $n \equiv 0 \bmod 4$, then every representation is spinorial by (17). If $n \equiv 1,2$ $\bmod 4$, then the adjoint representation is aspinorial, and if $n \equiv 3 \bmod 4$, then the second fundamental representation is aspinorial.

\section{TYPE $D_{n}$}

The simply connected group of type $D_{n}$ is $G_{\mathrm{sc}}=\operatorname{Spin}_{2 n}$. The center $Z$ of $G_{\mathrm{sc}}$ has order 4; in the notation of Section 2.4, it is generated by $c^{+}$when $n$ is odd, and generated by $c^{+}$and $z$ when $n$ is even.

Thus the groups of type $D_{n}$ for $n$ odd are $G_{\mathrm{sc}}$ and its quotients $\mathrm{SO}_{2 n}$ and $\mathrm{PSO}_{2 n}$. When $n$ is odd, the adjoint representation of $\mathrm{PSO}_{2 n}$ is aspinorial, which ends our investigation in this case. Henceforth in this section, we will assume that $n$ is even, and to ensure $\mathfrak{g}$ is simple we take $n>2$. (See Example 3 for $\mathrm{SO}_{4}$.)

For $n$ even, there are two more groups of type $D_{2 n}$ : the quotient $G_{2 n}^{+}$of $G_{\mathrm{sc}}$ by $\left\langle c^{+}\right\rangle$, and the quotient $G_{2 n}^{-}$of $G_{\mathrm{sc}}$ by $\left\langle-c^{+}\right\rangle$. Write $T_{2 n}=T_{V}$, where $V=F^{2 n}$, write $\widetilde{T}_{2 n}<G_{\mathrm{sc}}$ for its preimage, and write $T_{2 n}^{ \pm}$and $\bar{T}_{2 n}$ for the corresponding tori of $G_{2 n}^{ \pm}$and $\mathrm{PSO}_{2 n}$. The lattice of cocharacters corresponding to these quotients is depicted in Figure 1 . 
Recall that we identified $X_{*}\left(T_{2 n}\right)$ with $\mathbb{Z}^{n}$ in Section 2.4. Let

$$
Q=X_{*}\left(\widetilde{T}_{2 n}\right)=\left\{\left(b_{1}, \ldots, b_{n}\right) \in \mathbb{Z}^{n} \mid \sum b_{i} \text { is even }\right\} .
$$

Then

$$
X_{*}\left(T_{2 n}^{ \pm}\right)=Q+\mathbb{Z} \cdot \frac{1}{2}(1,1, \ldots, \pm 1)
$$

and

$$
X_{*}\left(\bar{T}_{2 n}\right)=\mathbb{Z}^{n}+\mathbb{Z} \cdot \frac{1}{2}(1,1, \ldots, 1) .
$$

All representations of groups of type $D_{n}$ are orthogonal. The standard representation $V_{0}$ of $\mathrm{SO}_{2 n}$ is evidently aspinorial. Thus, for the rest of this section we focus on the groups $\mathrm{PSO}_{2 n}$ and $G_{2 n}^{ \pm}$with $n$ even.

Tables 1 and 2 record the quantities $p(\underline{\nu}), \operatorname{dim} V_{\lambda}$, and $\chi_{\lambda}(C)$ that we need for our formulas. Here $\varpi_{k}=(\underbrace{1,1, \ldots, 1}_{k \text { times }}, 0, \ldots, 0)$ and $\varpi_{-}=(1,1, \ldots, 1,-1)$. (We

\begin{tabular}{|c|c|c|c|}
\hline$G$ & & $\underline{\nu}$ & $p(\underline{\nu})$ \\
\hline $\mathrm{SO}_{2 n}$ & & 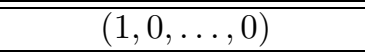 & $2 n-2$ \\
\hline $\mathrm{PSO}_{2 n}$ & $n \equiv 0 \bmod 4$ & $(1,0, \ldots, 0), \frac{1}{2}(1, \ldots, 1)$ & $2 n-2$ \\
\hline $\mathrm{PSO}_{2 n}$ & $n \equiv 2 \bmod 4$ & $(1,0, \ldots, 0), \frac{1}{2}(1, \ldots, 1)$ & $n-1$ \\
\hline$G_{2 n}^{ \pm}$ & & $\frac{1}{2}(1, \ldots, \pm 1)$ & $\left(\begin{array}{l}n \\
2\end{array}\right)$ \\
\hline
\end{tabular}
parametrize $X_{*}, X^{*}$ by $\mathbb{Z}^{n}$ as in Section 2.4.)

TABLE 1. Computing $p(\underline{\nu})$

TABLE 2. $\operatorname{dim} V_{\lambda}$ and $\chi_{\lambda}(C)$ for type $D_{n}, n$ even

\begin{tabular}{|c|c|c|}
\hline$\lambda$ & $\operatorname{dim} V_{\lambda}$ & $\chi_{\lambda}(C)$ \\
\hline$\varpi_{k}$ & $\left(\begin{array}{c}2 n \\
k\end{array}\right)$ & $\frac{k(2 n-k)}{4 n-4}$ \\
\hline$\frac{1}{2} \varpi_{-}$ & $2^{n-1}$ & $\frac{n(2 n-1)}{16(n-1)}$ \\
\hline$\frac{1}{2} \varpi_{n}$ & $2^{n-1}$ & $\frac{n(2 n-1)}{16(n-1)}$ \\
\hline$\varpi_{-}$ & $\frac{(2 n-1) !}{2^{n}}$ & $\frac{n^{2}}{4 n-4}$ \\
\hline
\end{tabular}

Remark 5. Let $n$ be odd. One has similarly $p\left(\nu_{0}\right)=n-2$ for $\mathrm{SO}_{n}$, with

$$
\nu_{0}(t)=\operatorname{diag}\left(t, 1, \ldots, 1, t^{-1}\right) .
$$

11.1. The case of $\mathrm{PSO}_{2 n}$. If $n \equiv 2 \bmod 4$, then the representation $\varphi$ of $\mathrm{PSO}_{2 n}$ on $\bigwedge^{2} V_{0}$ is aspinorial by Corollary [10, here $\operatorname{dyn}^{o}(\varphi)=2 n-2$ and $\breve{h}=2 n-2$, so

$$
p(\underline{\nu}) \cdot \frac{\operatorname{dyn}^{o}(\varphi)}{\check{h}}=n-1 .
$$


Let us assume for the rest of this section that $n$ is a multiple of 4 ; we will prove every orthogonal representation is spinorial in this case. We first consider $\varphi_{\lambda}$, with $\lambda$ in the set

$$
S_{0}=\left\{\varpi_{k}, \varpi_{-} \mid k \text { even, } 1 \leq k \leq n\right\} .
$$

Proposition 22. Each representation $\varphi_{\lambda}$ of $\mathrm{SO}_{2 n}$ with $\lambda \in S_{0}$ is spinorial.

Proof. Tables 1 and 2 give

$$
\begin{aligned}
q_{\varpi_{k}} & =\frac{1}{2} \frac{\left(\begin{array}{c}
2 n \\
k
\end{array}\right)}{\left(\begin{array}{c}
2 n \\
2
\end{array}\right)} \cdot k(2 n-k) \\
& =\left(\begin{array}{c}
2 n-2 \\
k-1
\end{array}\right) .
\end{aligned}
$$

Since $k$ is even, this is necessarily even, and we deduce that each $\varphi_{\varpi_{k}}$ is spinorial. Similarly

$$
q_{\varpi_{-}}=\frac{(2 n-2) ! n}{2^{n+1}} ;
$$

it is easy to see this is even for all $n$ divisible by 4 , thus $\varphi_{\varpi_{-}}$is spinorial.

These representations descend to $\mathrm{PSO}_{2 n}$, which are also spinorial by Corollary 9. Moreover, formulas (18) and (19) remain the same when computed for $\mathrm{PSO}_{2 n}$ (since the $p(\underline{\nu})$ are the same).

Let $S_{1}$ be the set

$S_{0} \cup\left\{\varpi_{k}+\varpi_{\ell} \mid k \equiv \ell \quad \bmod 2,1 \leq k, \ell \leq n\right\} \cup\left\{\varpi_{k}+\varpi_{-} \mid k\right.$ even $\} \cup\left\{2 \varpi_{-}\right\}$.

Note that $S_{1}$ has the following property. If $\lambda \in S_{1}$ and $\lambda^{\prime}$ is a dominant weight with $\lambda^{\prime} \prec \lambda$, then $\lambda^{\prime} \in S_{1}$.

Proposition 23. Each $\varphi_{\lambda}$ with $\lambda \in S_{1}$ is spinorial.

Proof. Suppose, by way of contradiction, that there are aspinorial $\lambda \in S_{1}$. Let $\lambda \in S_{1}$ be a $\prec$-minimal with $\varphi_{\lambda}$ aspinorial. We show below that for each of the three possibilities of $\lambda \in S_{1}$, the representation $\varphi_{\lambda}$ is spinorial, a contradiction.

If $\lambda \in S_{0}$ it is spinorial by Proposition 22, Otherwise $\lambda=\lambda_{1}+\lambda_{2}$ with $\lambda_{1}, \lambda_{2} \in S_{0}$, or $\lambda=\varpi_{k}+\varpi_{\ell}$ with $k, \ell$ odd.

In the first case, let $\Phi=\varphi_{\lambda_{1}} \otimes \varphi_{\lambda_{2}}$, which is spinorial by Proposition 22. By the property of $S_{1}$ mentioned above, we may apply Lemma 6(1) to deduce that $\varphi_{\lambda}$ is spinorial.

In the second case we have $\lambda=\varpi_{k}+\varpi_{\ell}$ with $k, \ell$ odd. Consider the representation $\Phi=\varphi_{\varpi_{k}} \otimes \varphi_{\varpi_{\ell}}$ of $\mathrm{SO}_{2 n}$. Applying equation (14) to the representations $\varphi_{\varpi_{k}}$ and $\varphi_{\varpi_{\ell}}$ of $\mathrm{SO}_{2 n}$ gives

$$
q_{\Phi}=\left(\begin{array}{c}
2 n \\
\ell
\end{array}\right)\left(\begin{array}{c}
2 n-2 \\
k-1
\end{array}\right)+\left(\begin{array}{c}
2 n \\
k
\end{array}\right)\left(\begin{array}{c}
2 n-2 \\
\ell-1
\end{array}\right) .
$$

Since this is even, $\Phi$ is a spinorial representation of $\mathrm{SO}_{2 n}$. By Corollary 9 it descends to a spinorial representation $\bar{\Phi}$ of $\mathrm{PSO}_{2 n}$.

Again, we may apply Lemma 6(1) to deduce that $\varphi_{\lambda}$ is spinorial. Thus in all cases we have a contradiction.

Theorem 8. When $n$ is divisible by 4 , every representation of $\mathrm{PSO}_{2 n}$ is spinorial. Proof. It is elementary to see that $S_{1}$ is a POSS. Thus the theorem follows by Propositions 14 and 23. 
11.2. The groups $G_{2 n}^{ \pm}$. Here $\check{h}=2 n-2$ and $p\left(\nu_{0}\right)=\left(\begin{array}{c}n \\ 2\end{array}\right)$, so by Corollary 10,

$$
\begin{aligned}
q_{\varphi}\left(\nu_{0}\right) & =\left(\begin{array}{l}
n \\
2
\end{array}\right) \cdot \frac{\operatorname{dyn}^{o}(\varphi)}{\check{h}} \\
& =\frac{n}{4} \cdot \operatorname{dyn}^{o}(\varphi) .
\end{aligned}
$$

If $n \equiv 2 \bmod 4$, then the representation $\varphi$ of $G_{2 n}^{ \pm}$on $\bigwedge^{2} V_{0}$ is aspinorial, since again $\operatorname{dyn}^{o}(\varphi)=2 n-2$. The half-spin representation $\left(\varphi_{\frac{1}{2} \varpi_{4}}, V_{\frac{1}{2} \varpi_{4}}\right)$ of $G_{8}^{+}$, and the half-spin representation $\left(\varphi_{\frac{1}{2} \varpi_{-}}, V_{\frac{1}{2} \varpi_{-}}\right)$of $G_{8}^{-}$are also aspinorial, since here $\operatorname{dyn}^{o}(\varphi)=1$.

Theorem 9. Suppose $n>4$ and a multiple of 4 . Then every orthogonal representation of $G_{2 n}^{+}$and $G_{2 n}^{-}$is spinorial.

Proof. If $n$ is a multiple of 8 , then the conclusion follows from (20).

If $n \equiv 4 \bmod 8$, then $\operatorname{ord}_{2}(p(\underline{\nu}))=1=\operatorname{ord}_{2}\left(p\left(\underline{\nu}^{\prime}\right)\right)$. Therefore we may apply Corollary 9 to see that a representation of $G_{2 n}^{ \pm}$which descends to $\mathrm{PSO}_{2 n}$ is spinorial iff it was originally spinorial. Thus by Theorem 8 , all such representations of $G_{2 n}^{ \pm}$ are spinorial.

However there are representations of $G_{2 n}^{ \pm}$which don't descend, so we must enlarge our POSS. Let $S^{+}=S_{1} \cup\left\{\frac{1}{2} \varpi_{n}\right\}$ and $S^{-}=S_{1} \cup\left\{\frac{1}{2} \varpi_{-}\right\}$. Then $S^{ \pm}$is a POSS for $G^{ \pm}$. By (20), we have

$$
q_{\frac{1}{2} \varpi_{n}}=q_{\frac{1}{2} \varpi_{-}}=n 2^{n-6},
$$

which is certainly even. Thus for each $\lambda \in S^{ \pm}$, the representation $V_{\lambda}$ of $G_{2 n}^{ \pm}$is spinorial. The conclusion then follows by Proposition 14.

11.3. Summary for groups of type $D_{n}$. Let $n>2$ be a positive integer. From the above we know:

- The standard representation of $\mathrm{SO}_{2 n}$ is aspinorial.

- If $n$ is a multiple of 4 , then every representation of $\mathrm{PSO}_{2 n}$ is spinorial.

- If $n$ is odd, then the adjoint representation of $\mathrm{PSO}_{2 n}$ is aspinorial.

- If $n \equiv 2 \bmod 4$, then the representations of $\mathrm{PSO}_{2 n}$ and $G_{2 n}^{ \pm}$on $\bigwedge^{2} V_{0}$ are aspinorial.

- The half-spin representation $\varphi_{\frac{1}{2} \varpi_{4}}$ of $G_{8}^{+}$and the half-spin representation $\varphi_{\frac{1}{2} \varpi_{-}}$of $G_{8}^{-}$are aspinorial.

- For $n>4$ a multiple of 4 , all representations of $G_{2 n}^{+}$and $G_{2 n}^{-}$are spinorial.

\section{SumMARY FOR SIMPLE $\mathfrak{g}$}

Here is a list of all $G$ with simple $\mathfrak{g}$, with the property that all orthogonal representations of $G$ are spinorial:

- All $G$ whose fundamental group has odd order.

- All $\mathrm{SL}_{n} / \mu_{d}$, when $n / d$ is even, except when $n$ is a power of 2 and $d=n / 2$.

- $\mathrm{Sp}_{2 n} / \pm 1$, when $n$ is a multiple of 4 .

- The groups $\mathrm{PSO}_{n}$, when $n$ is a multiple of 8 .

- The groups $G_{2 n}^{ \pm}$, when $n>4$ is a multiple of 4 . 
For the reader's convenience, we recall the $G$ whose fundamental groups have odd order:

- Simply connected $G$.

- $\mathrm{SL}_{n} / \mu_{d}$ with $d$ odd.

- The adjoint group of type $E_{6}$.

Aspinorial representations for most groups not on this list have already been mentioned. To finish, we remark that the standard representation of an odd orthogonal group is aspinorial, and the adjoint representation of the adjoint group of type $E_{7}$ is aspinorial.

\section{Periodicity}

For the irreducible orthogonal representations $\varphi_{\lambda}$, our lifting criterion amounts to determining the parity of one or more $q_{\lambda}(\nu)$, each an integer-valued polynomial function of $\lambda$. As we explain in this section, this entails a certain periodicity of the spinorial highest weights in the character lattice.

13.1. Polynomials with integer values. Let $V$ be a finite-dimensional rational vector space, $V^{*}$ its dual, $L$ a lattice in $V$, and $L^{\vee}$ the dual lattice in $V^{*}$. Recall that $L^{\vee}$ is the $\mathbb{Z}$-module of $\mathbb{Q}$-linear maps $f: V \rightarrow \mathbb{Q}$ so that $f(L) \subseteq \mathbb{Z}$. Denote by $\left(\begin{array}{c}L^{\vee} \\ \mathbb{Z}\end{array}\right)$ the $\mathbb{Z}$-algebra of polynomial functions on $V$ which take integer values on $L$. Given $f \in L^{\vee}$, and $n \in \mathbb{N}$, define $\left(\begin{array}{l}f \\ n\end{array}\right) \in\left(\begin{array}{c}L^{\vee} \\ \mathbb{Z}\end{array}\right)$ by the prescription

$$
\left(\begin{array}{c}
f \\
n
\end{array}\right): x \mapsto\left(\begin{array}{c}
f(x) \\
n
\end{array}\right)=\frac{f(x)(f(x)-1) \cdots(f(x)-n+1)}{n !}
$$

for $x \in L$.

Proposition 24. The $\mathbb{Z}$-algebra $\left(\begin{array}{c}L^{\vee} \\ \mathbb{Z}\end{array}\right)$ is generated by the $\left(\begin{array}{l}f \\ n\end{array}\right)$ for $f \in L^{\vee}$ and $n \in \mathbb{N}$. If $\left\{f_{1}, \ldots, f_{r}\right\}$ is a $\mathbb{Z}$-basis of $L^{\vee}$, then the products

$$
\left(\begin{array}{c}
f_{1} \\
n_{1}
\end{array}\right) \cdots\left(\begin{array}{c}
f_{r} \\
n_{r}
\end{array}\right)
$$

where $n_{1}, \ldots, n_{r} \in \mathbb{N}$, form a basis of the $\mathbb{Z}$-module $\left(\begin{array}{c}L^{\vee} \\ \mathbb{Z}\end{array}\right)$.

Proof. See Proposition 2 in Bou05, Chapter 8, Section 12, no. 4.

Given a basis of $V$, we can form the set $C$ of its nonnegative linear combinations. Call $C$ a "full polyhedral cone" if it arises in this way, and write $L^{+}=L \cap C$.

Proposition 25. Suppose $f$ is a polynomial map from $V$ to $\mathbb{Q}$ that takes integer values on $L^{+}$. Then $f \in\left(\begin{array}{c}L^{\vee} \\ \mathbb{Z}\end{array}\right)$.

Proof. We omit the elementary proof (see [Jos18]) of the following lemma.

Lemma 7. Suppose that $V$ is a finite-dimensional rational vector space, that $C$ is a full polyhedral cone in $V$, and that $L \subset V$ is a lattice. Let $p \in L$. Then:

(1) $C \cap(p+C)$ is a translation of $C$.

(2) The intersection $L \cap C \cap(p+C)$ is nonempty.

(3) Suppose $p^{\prime}$ is in the above intersection, and write $v=p^{\prime}-p$. Then $p+n v \in$ $L \cap C \cap(p+C)$ for all positive integers $n$. 
Continuing with the proof of the proposition, let $\ell \in L$; we must show that $f(\ell) \in \mathbb{Z}$. By the lemma there is a $v \in L$ so that $\ell+n v \in L^{+}$for all positive integers $n$. For $x \in \mathbb{Z}$, put $g(x)=f(\ell+x v)$. Then $g \in \mathbb{Q}[x]$, and by hypothesis it takes integer values on positive integers. It is elementary to see that such a polynomial takes integer values at all integers, and in particular $g(0)=f(\ell) \in \mathbb{Z}$.

Lemma 8. Fix an integer $n \geq 1$ and put $k=\left[\log _{2} n\right]+1$. Then $\left(\begin{array}{c}a+2^{k} \\ n\end{array}\right) \equiv\left(\begin{array}{l}a \\ n\end{array}\right)$ mod 2 for every integer $a \geq 1$.

Proof. This follows from the Lucas congruence (see, e.g., [Sta12]).

Proposition 26. Let $f \in\left(\begin{array}{c}L^{\vee} \\ \mathbb{Z}\end{array}\right)$. Then there is a $k \in \mathbb{N}$ so that for all $x, y \in L$ we have

$$
f\left(x+2^{k} y\right) \equiv f(x) \quad \bmod 2 .
$$

Proof. By Proposition 24, there are $f_{1}, \ldots, f_{r} \in L^{\vee}$, integers $n_{1}, \ldots, n_{r}$, and a polynomial $g \in \mathbb{Z}\left[x_{1}, \ldots, x_{r}\right]$ so that

$$
f=g\left(\left(\begin{array}{c}
f_{1} \\
n_{1}
\end{array}\right), \ldots,\left(\begin{array}{c}
f_{r} \\
n_{r}
\end{array}\right)\right) .
$$

Let $k_{i}=\left[\log _{2} n_{i}\right]+1$; by Lemma 8 we have

$$
\left(\begin{array}{c}
f_{i} \\
n_{i}
\end{array}\right)\left(x+2^{k_{i}} y\right) \equiv\left(\begin{array}{c}
f_{i} \\
n_{i}
\end{array}\right)(x) \bmod 2
$$

for all $x, y \in L$. If we put $k=\max \left(k_{1}, \ldots, k_{r}\right)$ we obtain the proposition.

13.2. Example: Parity of dimensions. To illustrate the above, let $G$ be connected reductive with notation as before. Take $L=X^{*}(T) \subset V=X^{*}(T) \otimes \mathbb{Q} \hookrightarrow \mathfrak{t}^{*}$. Define $f: \mathfrak{t}^{*} \rightarrow F$ by

$$
f(\lambda)=\frac{d_{\lambda+\delta}}{d_{\delta}}=\operatorname{dim} V_{\lambda} .
$$

From Propositions 25] and 26 we deduce the following corollary.

Corollary 12. With notation as above:

(1) $f(\lambda) \in \mathbb{Z}$ for all $\lambda \in X^{*}(T)$; equivalently $f \in\left(\begin{array}{c}X_{*}(T) \\ \mathbb{Z}\end{array}\right)$.

(2) There is a $k \in \mathbb{N}$ so that $f\left(\lambda_{0}+2^{k} \lambda\right) \equiv f\left(\lambda_{0}\right) \bmod 2$ for all $\lambda_{0}, \lambda \in X^{*}(T)$.

13.3. Proof of Theorem 2], We continue with $G$ connected reductive.

If $\mathfrak{g}$ is simple put

Then:

$$
\eta_{\underline{\nu}}(\lambda)=p(\underline{\nu}) \cdot \frac{\operatorname{dim} V_{\lambda} \cdot \chi_{\lambda}(C)}{\operatorname{dim} \mathfrak{g}}
$$

(1) $\eta_{\underline{\nu}}$ is a polynomial in $\lambda$,

(2) $\eta_{\underline{\nu}}(\lambda) \in \mathbb{Z}$ for $\lambda \in X_{\text {sd }}^{+}$, and

(3) $\varphi_{\lambda}$ is spinorial iff $\eta_{\underline{\nu}}(\lambda)$ is even.

If $\mathfrak{g}$ is not necessarily simple, we may instead put

$$
\eta_{\underline{\nu}}(\lambda)=1+\prod_{\nu \in \underline{\nu}}\left(q_{\lambda}(\nu)-1\right),
$$

and the same three properties hold. From Propositions 25] and 26] we deduce the following corollary. 
Corollary 13. With notation as above:

(1) $\eta_{\underline{\nu}}(\lambda) \in \mathbb{Z}$ for all $\lambda \in X_{\text {orth }} ;$ equivalently $\eta_{\underline{\nu}} \in\left(\begin{array}{c}X_{\text {orth }}^{\vee} \\ \mathbb{Z}^{\circ}\end{array}\right)$.

(2) There is a $k \in \mathbb{N}$ so that $\eta_{\underline{\nu}}\left(\lambda_{0}+2^{k} \lambda\right) \equiv \eta_{\underline{\nu}}\left(\lambda_{0}\right) \bmod 2$ for all $\lambda_{0}, \lambda \in X_{\text {orth }}$.

Theorem 2 in the introduction follows from this. If we put $L^{+}=2^{k} X_{\text {orth }}^{+}$, then the theorem says that the set of spinorial highest weights is stable under addition from $L^{+}$. Since the index $\left[X_{\text {orth }}: 2^{k} X_{\text {orth }}\right]$ is finite, the determination of the full set of spinorial weights amounts to a finite computation.

The problem of finding the exact largest lattice $L \subseteq X_{\text {orth }}$ so that the spinorialities of $\varphi_{\lambda_{0}}$ and $\varphi_{\lambda_{0}+\ell}$ agree for all $\lambda \in X_{\text {orth }}^{+}$and $\ell \in L^{+}$seems interesting, as does the problem of determining the proportion of spinorial irreducible representations. We do not settle these questions here, but see the next section for $\mathrm{PGL}_{2}$ and $\mathrm{SO}_{4}$, and [Jos18] for more examples.

13.4. Examples. Let us examine $G=\mathrm{PGL}_{2}$ more closely. We have $X^{*}(T)=$ $X_{\text {sd }}=X_{\text {orth }}$. For integers $j \geq 0$ define $\lambda_{j} \in X^{*}(T)$ by

$$
\lambda_{j}\left(\left(\begin{array}{ll}
a & \\
& b
\end{array}\right)\right)=\left(a b^{-1}\right)^{j} .
$$

Then $\operatorname{dim} V_{\lambda_{j}}=2 j+1$ and $\chi_{\lambda_{j}}(C)=\frac{1}{2}\left(j^{2}+j\right)$, so $\varphi_{\lambda_{j}}$ is spinorial iff

$$
\frac{j(j+1)(2 j+1)}{2}
$$

is even. Equivalently, $j \equiv 0,3 \bmod 4$. We may therefore take $k=2$ in Theorem 2,

As a second example, recall the representations $V_{a, b}$ of $\mathrm{SO}_{4}$ from Example 3 . If we put

$$
F(a, b)=\frac{1}{4}\left((b+1)\left(\begin{array}{c}
a+2 \\
3
\end{array}\right)+(a+1)\left(\begin{array}{c}
b+2 \\
3
\end{array}\right)\right),
$$

then $V_{a, b}$ is spinorial iff $F(a, b)$ is even. It is elementary to see that $F(a+8 i, b+8 j) \equiv$ $F(a, b) \bmod 2$ for integers $i, j$. In particular we may take $k=3$ in Theorem 2

\section{Reduction to ALGEBRAiCALLy CLOSED FIELDS}

For this section, $G$ is a connected reductive group defined over a field $F$ of characteristic 0 , not necessarily algebraically closed. Let $V$ be a quadratic vector space over $F$, and $\varphi: G \rightarrow \mathrm{SO}(V)$ a morphism defined over $F$. The isogeny $\rho: \operatorname{Spin}(V) \rightarrow \mathrm{SO}(V)$ is also defined over $F$. By extending scalars to the algebraic closure $\bar{F}$ of $F$, we may use the rest of this paper to determine whether there exists a lift $\hat{\varphi}: G \rightarrow \operatorname{Spin}(V)$ of $\varphi$ defined over $\bar{F}$.

Lemma 9. If $\hat{\varphi}: G \rightarrow \operatorname{Spin}(V)$ is a lift defined over $\bar{F}$, then it arises from a lift defined over $F$.

Proof. The Galois group acts by Zariski-continuous automorphisms on the $\bar{F}$-points of $G$ and $\operatorname{Spin}(V)$. We must show that for every $\sigma \in \operatorname{Gal}(F)$ and $x \in G(\bar{F})$, we have ${ }^{\sigma} \hat{\varphi}(x)=\hat{\varphi}\left({ }^{\sigma} x\right)$. Since $\rho$ and $\varphi$ are defined over $F$, the identity $\rho(\hat{\varphi}(x))=\varphi(x)$ implies that

$$
\rho\left(\hat{\varphi}(x)^{-1} \cdot \sigma^{-1} \hat{\varphi}\left({ }^{\sigma} x\right)\right)=1 .
$$

Thus the argument of $\rho$ above gives a Zariski-continuous map $G(\bar{F}) \rightarrow$ ker $\rho$. Since $G$ is connected and $\operatorname{ker} \rho$ is discrete, it must be that $\hat{\varphi}(x)=\sigma^{-1} \hat{\varphi}\left({ }^{\sigma} x\right)$, and the lemma follows. 
Therefore: the $F$-representation $\varphi$ is spinorial iff its extension to $\bar{F}$-points is spinorial.

\section{ACKNOWLEDGMEnTs}

We would like to thank Dipendra Prasad for his interest and useful conversations, and Raghuram and Jeff Adler for helpful suggestions. We would like to thank the referee for useful comments and suggestions on an earlier version of this manuscript.

\section{REFERENCES}

[Ada69] J. Frank Adams, Lectures on Lie groups, W. A. Benjamin, Inc., New York-Amsterdam, 1969. MR0252560

[Bou02] Nicolas Bourbaki, Lie groups and Lie algebras. Chapters 4-6, Elements of Mathematics (Berlin), Springer-Verlag, Berlin, 2002. Translated from the 1968 French original by Andrew Pressley. MR.1890629

[Bou05] Nicolas Bourbaki, Lie groups and Lie algebras. Chapters 7-9, Elements of Mathematics (Berlin), Springer-Verlag, Berlin, 2005. Translated from the 1975 and 1982 French originals by Andrew Pressley. MR2109105

[Dyn52] E. B. Dynkin, Semisimple subalgebras of semisimple Lie algebras (Russian), Mat. Sbornik N.S. 30(72) (1952), 349-462 (3 plates). MR0047629

[Dyn00] E. B. Dynkin, Selected papers of E. B. Dynkin with commentary, American Mathematical Society, Providence, RI; International Press, Cambridge, MA, 2000. Edited by A. A. Yushkevich, G. M. Seitz and A. L. Onishchik. MR.1757976

[FH91] William Fulton and Joe Harris, Representation theory: A first course; Readings in Mathematics, Graduate Texts in Mathematics, vol. 129, Springer-Verlag, New York, 1991. MR.1153249

[GW09] Roe Goodman and Nolan R. Wallach, Symmetry, representations, and invariants, Graduate Texts in Mathematics, vol. 255, Springer, Dordrecht, 2009. MR.2522486

[Jan03] Jens Carsten Jantzen, Representations of algebraic groups, 2nd ed., Mathematical Surveys and Monographs, vol. 107, American Mathematical Society, Providence, RI, 2003. MR.2015057

[Jos18] R. Joshi, Spinorial representations of Lie groups, PhD thesis, Indian Institute of Science Education and Research, Pune, 2018.

[Jr.08] Alexander Kirillov Jr., An introduction to Lie groups and Lie algebras, Cambridge Studies in Advanced Mathematics, vol. 113, Cambridge University Press, Cambridge, 2008. MR.2440737

[Kac90] Victor G. Kac, Infinite-dimensional Lie algebras, 3rd ed., Cambridge University Press, Cambridge, 1990. MR.1104219

[Kos76] Bertram Kostant, On Macdonald's $\eta$-function formula, the Laplacian and generalized exponents, Advances in Math. 20 (1976), no. 2, 179-212, DOI 10.1016/0001-8708(76)901869. MR 485661

[PR95] Dipendra Prasad and Dinakar Ramakrishnan, Lifting orthogonal representations to spin groups and local root numbers, Proc. Indian Acad. Sci. Math. Sci. 105 (1995), no. 3, 259-267, DOI 10.1007/BF02837191. MR 1369731

[Sam90] Hans Samelson, Notes on Lie algebras, 2nd ed., Universitext, Springer-Verlag, New York, 1990. MR 1056083

[Spa66] Edwin H. Spanier, Algebraic topology, McGraw-Hill Book Co., New York-Toronto, Ont.London, 1966. MR0210112

[Spr79] T. A. Springer, Reductive groups, Automorphic forms, representations and $L$-functions (Proc. Sympos. Pure Math., Oregon State Univ., Corvallis, Ore., 1977), Proc. Sympos. Pure Math., XXXIII, Amer. Math. Soc., Providence, R.I., 1979, pp. 3-27. MR546587

[Spr98] T. A. Springer, Linear algebraic groups, 2nd ed., Progress in Mathematics, vol. 9, Birkhäuser Boston, Inc., Boston, MA, 1998. MR1642713

[Sta12] Richard P. Stanley, Enumerative combinatorics. Volume 1, 2nd ed., Cambridge Studies in Advanced Mathematics, vol. 49, Cambridge University Press, Cambridge, 2012. $\operatorname{MR} 2868112$ 
[SV00] Tonny A. Springer and Ferdinand D. Veldkamp, Octonions, Jordan algebras and exceptional groups, Springer Monographs in Mathematics, Springer-Verlag, Berlin, 2000. MR.1763974

Bhaskaracharya Pratishthana, 56/14, Erandavane, Damle Path, off Law College Road, Pune-411004, Maharashtra, India; and Indian Institute of Science Education and Research, Pune-411021, India

Email address: rohitsj@students.iiserpune.ac.in

Email address: rohitsj2004@gmail.com

Indian Institute of Science Education and Research, Pune-411021, India

Email address: sspallone@gmail.com 